\title{
Measurement of beauty photoproduction near threshold using di-electron events with the H1 detector at HERA
}

The H1 Collaboration

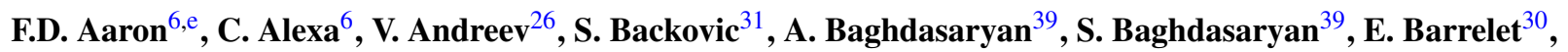
W. Bartel ${ }^{12}$, K. Begzsuren ${ }^{36}$, A. Belousov ${ }^{26}$, P. Belov ${ }^{12}$, J.C. Bizot ${ }^{28}$, V. Boudry ${ }^{29}$, I. Bozovic-Jelisavcic $^{2}$, J. Bracinik ${ }^{3}$, G. Brandt $^{12}$, M. Brinkmann ${ }^{12}$, V. Brisson ${ }^{28}$, D. Britzger ${ }^{12}$, D. Bruncko ${ }^{17}$, A. Bunyatyan ${ }^{14,39}$, A. Bylinkin ${ }^{25}$, L. Bystritskaya ${ }^{25}$, A.J. Campbell ${ }^{12}$, K.B. Cantun Avila ${ }^{23}$, F. Ceccopieri ${ }^{4,5}$, K. Cerny ${ }^{33}$, V. Cerny ${ }^{17}$, V. Chekelian ${ }^{27}$, J.G. Contreras ${ }^{23}$, J.A. Coughlan ${ }^{7}$, J. Cvach $^{32}$, J.B. Dainton ${ }^{19}$, K. Daum ${ }^{38, b}$, B. Delcourt ${ }^{28}$, J. Delvax $^{4,5}$, E.A. De Wolf ${ }^{4,5}$, C. Diaconu ${ }^{22}$, M. Dobre ${ }^{13, g, h}$, V. Dodonov ${ }^{14}$, A. Dossanov ${ }^{13,27}$, A. Dubak ${ }^{31}$, G. Eckerlin $^{12}$, S. Egli $^{37}$, A. Eliseev $^{26}$, E. Elsen ${ }^{12}$, L. Favart ${ }^{4,5}$, A. Fedotov ${ }^{25}$, R. Felst ${ }^{12}$, J. Feltesse ${ }^{11}$, J. Ferencei ${ }^{17}$, D.-J. Fischer ${ }^{12}$, M. Fleischer ${ }^{12}$, A. Fomenko ${ }^{26}$, E. Gabathuler ${ }^{19}$, J. Gayler ${ }^{12}$, S. Ghazaryan ${ }^{12}$, A. Glazov ${ }^{12}$ L. Goerlich $^{8}$, N. Gogitidze ${ }^{26}$, M. Gouzevitch ${ }^{12, c}$, C. Grab ${ }^{41}$, A. Grebenyuk ${ }^{12}$, T. Greenshaw ${ }^{19}$, G. Grindhammer ${ }^{27}$, S. Habib $^{12}$, D. Haidt ${ }^{12}$, R.C.W. Henderson ${ }^{18}$, E. Hennekemper ${ }^{16}$, H. Henschel ${ }^{40}$, M. Herbst ${ }^{16}$, G. Herrera ${ }^{24}$, M. Hildebrandt ${ }^{37}$, K.H. Hiller ${ }^{40}$, D. Hoffmann ${ }^{22}$, R. Horisberger ${ }^{37}$, T. Hreus ${ }^{4,5}$, F. Huber ${ }^{15}$, M. Jacquet ${ }^{28}$, X. Janssen ${ }^{4,5}$, L. Jönsson $^{21}$, A.W. Jung ${ }^{16, k}$, H. Jung ${ }^{12,4,5}$, M. Kapichine ${ }^{10}$, I.R. Kenyon ${ }^{3}$, C. Kiesling ${ }^{27}$, M. Klein ${ }^{19}$, C. Kleinwort ${ }^{12}$, R. Kogler $^{13}$, P. Kostka ${ }^{40}$, M. Krämer ${ }^{12}$, J. Kretzschmar ${ }^{19}$, K. Krüger ${ }^{16, a}$, M.P.J. Landon ${ }^{20}$, W. Lange , G. Laštovička-Medin $^{31}$, P. Laycock ${ }^{19}$, A. Lebedev ${ }^{26}$, V. Lendermann ${ }^{16}$, S. Levonian ${ }^{12}$, K. Lipka ${ }^{12, g}$, B. List ${ }^{12}$, J. List ${ }^{12}$, B. Lobodzinski ${ }^{12}$, R. Lopez-Fernandez ${ }^{24}$, V. Lubimov ${ }^{25}$, E. Malinovski ${ }^{26}$, H.-U. Martyn ${ }^{1}$, S.J. Maxfield ${ }^{19}$, A. Mehta ${ }^{19}$, A.B. Meyer ${ }^{12}$, H. Meyer $^{38}$, J. Meyer $^{12}$, S. Mikocki ${ }^{8}$, I. Milcewicz-Mika ${ }^{8}$, F. Moreau $^{29}$, A. Morozov ${ }^{10}$, J.V. Morris ${ }^{7}$, K. Müller ${ }^{42}$, Th. Naumann ${ }^{40}$, P.R. Newman ${ }^{3}$, C. Niebuhr ${ }^{12}$, D. Nikitin ${ }^{10}$, G. Nowak $^{8}$, K. Nowak ${ }^{13}$, B. Olivier ${ }^{27}$, J.E. Olsson ${ }^{12}$, D. Ozerov ${ }^{12}$, P. Pahl ${ }^{12}$, V. Palichik ${ }^{10}$, M. Pandurovic ${ }^{2}$, C. Pascaud ${ }^{28}$, G.D. Patel ${ }^{19}$, E. Perez ${ }^{11, \text {, }}$, A. Petrukhin ${ }^{12}$, I. Picuric $^{31}$, H. Pirumov ${ }^{15}$, D. Pitzl ${ }^{12}$, R. Plačakyté ${ }^{12}$, B. Pokorny ${ }^{33}$, R. Polifka ${ }^{33, i}$, B. Povh ${ }^{14}$, V. Radescu ${ }^{12}$, N. Raicevic $^{31}$, T. Ravdandorj $^{36}$, P. Reimer ${ }^{32}$, E. Rizvi ${ }^{20}$, P. Robmann ${ }^{42}$, R. Roosen ${ }^{4,5}$, A. Rostovtsev $^{25}$, M. Rotaru $^{6}$, J.E. Ruiz Tabasco ${ }^{23}$, S. Rusakov ${ }^{26}$, D. Š́lek ${ }^{33}$, D.P.C. Sankey ${ }^{7}$, M. Sauter ${ }^{15}$, E. Sauvan ${ }^{22, j}$, S. Schmitt ${ }^{12}$, L. Schoeffel $^{11}$, A. Schöning ${ }^{15}$, H.-C. Schultz-Coulon ${ }^{16}$, F. Sefkow ${ }^{12}$, L.N. Shtarkov ${ }^{26}$, S. Shushkevich ${ }^{12}$, T. Sloan ${ }^{18}$, Y. Soloviev ${ }^{12,26}$, P. Sopicki ${ }^{8}$, D. South ${ }^{12}$, V. Spaskov ${ }^{10}$, A. Specka ${ }^{29}$, Z. Staykova ${ }^{4,5}$, M. Steder ${ }^{12}$, B. Stella ${ }^{34}$,

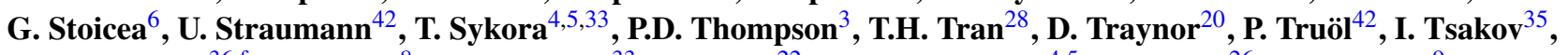
B. Tseepeldorj ${ }^{36, f}$, J. Turnau ${ }^{8}$, A. Valkárová ${ }^{33}$, C. Vallée $^{22}$, P. Van Mechelen ${ }^{4,5}$, Y. Vazdik ${ }^{26}$, D. Wegener $^{9}$,

E. Wünsch ${ }^{12}$, J. Žáček ${ }^{33}$, J. Zálešák ${ }^{32}$, Z. Zhang ${ }^{28}$, A. Zhokin ${ }^{25}$, R. Žlebčík ${ }^{33}$, H. Zohrabyan $^{39}$, F. Zomer $^{28}$

${ }^{1}$ I. Physikalisches Institut der RWTH, Aachen, Germany

${ }^{2}$ Vinca Institute of Nuclear Sciences, University of Belgrade, 1100 Belgrade, Serbia

${ }^{3}$ School of Physics and Astronomy, University of Birmingham, Birmingham, $\mathrm{UK}^{\mathrm{m}}$

${ }^{4}$ Inter-University Institute for High Energies ULB-VUB, Brussels, Belgium ${ }^{\text {n }}$

${ }^{5}$ Universiteit Antwerpen, Antwerpen, Belgium ${ }^{\text {n }}$

${ }^{6}$ National Institute for Physics and Nuclear Engineering (NIPNE), Bucharest, Romaniav

${ }^{7}$ Rutherford Appleton Laboratory, STFC, Didcot, Oxfordshire, $\mathrm{UK}^{\mathrm{m}}$

${ }^{8}$ Institute for Nuclear Physics, Cracow, Poland ${ }^{\circ}$

${ }^{9}$ Institut für Physik, TU Dortmund, Dortmund, Germany ${ }^{1}$

${ }^{10}$ Joint Institute for Nuclear Research, Dubna, Russia

${ }^{11} \mathrm{CEA}, \mathrm{DSM} / \mathrm{Irfu}, \mathrm{CE}-$ Saclay, Gif-sur-Yvette, France

${ }^{12}$ DESY, Hamburg, Germany

${ }^{13}$ Institut für Experimentalphysik, Universität Hamburg, Hamburg, Germany ${ }^{1}$

${ }^{14}$ Max-Planck-Institut für Kernphysik, Heidelberg, Germany

${ }^{15}$ Physikalisches Institut, Universität Heidelberg, Heidelberg, Germany ${ }^{1}$

${ }^{16}$ Kirchhoff-Institut für Physik, Universität Heidelberg, Heidelberg, Germany ${ }^{1}$

${ }^{17}$ Institute of Experimental Physics, Slovak Academy of Sciences, Košice, Slovak Republic ${ }^{p}$

${ }^{18}$ Department of Physics, University of Lancaster, Lancaster, UK $^{\mathrm{m}}$

${ }^{19}$ Department of Physics, University of Liverpool, Liverpool, $\mathrm{UK}^{\mathrm{m}}$

${ }^{20}$ School of Physics and Astronomy, Queen Mary, University of London, London, $\mathrm{UK}^{\mathrm{m}}$ 
${ }^{21}$ Physics Department, University of Lund, Lund, Sweden ${ }^{\mathrm{q}}$

${ }^{22}$ CPPM, Aix-Marseille Univ, CNRS/IN2P3, 13288 Marseille, France

${ }^{23}$ Departamento de Fisica Aplicada, CINVESTAV, Mérida, Yucatán, Mexico ${ }^{t}$

${ }^{24}$ Departamento de Fisica, CINVESTAV IPN, México City, Mexico ${ }^{t}$

${ }^{25}$ Institute for Theoretical and Experimental Physics, Moscow, Russia ${ }^{\mathrm{u}}$

${ }^{26}$ Lebedev Physical Institute, Moscow, Russia

${ }^{27}$ Max-Planck-Institut für Physik, München, Germany

${ }^{28}$ LAL, Université Paris-Sud, CNRS/IN2P3, Orsay, France

${ }^{29}$ LLR, Ecole Polytechnique, CNRS/IN2P3, Palaiseau, France

${ }^{30}$ LPNHE, Université Pierre et Marie Curie Paris 6, Université Denis Diderot Paris 7, CNRS/IN2P3, Paris, France

${ }^{31}$ Faculty of Science, University of Montenegro, Podgorica, Montenegrow

${ }^{32}$ Institute of Physics, Academy of Sciences of the Czech Republic, Praha, Czech Republic ${ }^{\mathrm{r}}$

${ }^{33}$ Faculty of Mathematics and Physics, Charles University, Praha, Czech Republic ${ }^{\mathrm{r}}$

${ }^{34}$ Dipartimento di Fisica Università di Roma Tre and INFN Roma 3, Roma, Italy

${ }^{35}$ Institute for Nuclear Research and Nuclear Energy, Sofia, Bulgaria

${ }^{36}$ Institute of Physics and Technology of the Mongolian Academy of Sciences, Ulaanbaatar, Mongolia

${ }^{37}$ Paul Scherrer Institut, Villigen, Switzerland

${ }^{38}$ Fachbereich C, Universität Wuppertal, Wuppertal, Germany

${ }^{39}$ Yerevan Physics Institute, Yerevan, Armenia

${ }^{40}$ DESY, Zeuthen, Germany

${ }^{41}$ Institut für Teilchenphysik, ETH, Zürich, Switzerland ${ }^{\mathrm{s}}$

${ }^{42}$ Physik-Institut der Universität Zürich, Zürich, Switzerland ${ }^{\mathrm{s}}$

Received: 19 June 2012 / Revised: 10 August 2012 / Published online: 3 October 2012

(C) The Author(s) 2012. This article is published with open access at Springerlink.com

\begin{abstract}
The cross section for $e p \rightarrow e b \bar{b} X$ in photoproduction is measured with the $\mathrm{H} 1$ detector at the $e p$-collider HERA. The decay channel $b \bar{b} \rightarrow e e X^{\prime}$ is selected by identifying the semi-electronic decays of the $b$-quarks. The total production cross section is measured in the kinematic range given by the photon virtuality $Q^{2} \leq 1 \mathrm{GeV}^{2}$, the inelasticity $0.05 \leq y \leq 0.65$ and the pseudorapidity of the $b$-quarks $|\eta(b)|,|\eta(\bar{b})| \leq 2$. The differential production cross section is measured as a function of the average transverse momentum of the beauty quarks $\left\langle P_{T}(b)\right\rangle$ down to the threshold. The
\end{abstract}

a e-mail: kruegerk@mail.desy.de

${ }^{b}$ Also at Rechenzentrum, Universität Wuppertal, Wuppertal, Germany.

${ }^{\mathrm{c}}$ Also at IPNL, Université Claude Bernard Lyon 1, CNRS/IN2P3, Villeurbanne, France.

${ }^{\mathrm{d}}$ Also at CERN, Geneva, Switzerland.

${ }^{\mathrm{e}}$ Also at Faculty of Physics, University of Bucharest, Bucharest, Romania.

${ }^{\mathrm{f}}$ Also at Ulaanbaatar University, Ulaanbaatar, Mongolia.

${ }^{\mathrm{g}}$ Supported by the Initiative and Networking Fund of the Helmholtz Association (HGF) under the contract VH-NG-401.

${ }^{\mathrm{h}}$ Absent on leave from NIPNE-HH, Bucharest, Romania.

${ }^{\mathrm{i}}$ Also at Department of Physics, University of Toronto, Toronto, Ontario, Canada M5S 1 A7.

${ }^{\mathrm{j}}$ Also at LAPP, Université de Savoie, CNRS/IN2P3, Annecy-le-Vieux, France.

${ }^{k}$ Now at Fermi National Accelerator Laboratory, Batavia, Illinois 60510, USA.

${ }^{1}$ Supported by the Bundesministerium für Bildung und Forschung, FRG, under contract numbers 05H09GUF, 05H09VHC, 05H09VHF, 05H16PEA. results are compared to next-to-leading-order QCD predictions.

\section{Introduction}

In $e p$ collisions at HERA beauty quarks are mainly produced as $b \bar{b}$ pairs via the fusion of a quasi-real photon emitted by the incoming electron (or positron) and a gluon of the proton as depicted in Fig. 1a. This process is referred to as direct or pointlike and can be calculated using perturbative

\footnotetext{
${ }^{\mathrm{m}}$ Supported by the UK Science and Technology Facilities Council, and formerly by the UK Particle Physics and Astronomy Research Council.

${ }^{n}$ Supported by FNRS-FWO-Vlaanderen, IISN-IIKW and IWT and by Interuniversity Attraction Poles Programme, Belgian Science Policy.

${ }^{\circ}$ Partially Supported by Polish Ministry of Science and Higher Education, grant DPN/N168/DESY/2009.

${ }^{\mathrm{p}}$ Supported by VEGA SR grant no. 2/7062/27.

${ }^{\mathrm{q}}$ Supported by the Swedish Natural Science Research Council.

${ }^{\mathrm{r}}$ Supported by the Ministry of Education of the Czech Republic under the projects LC527, INGO-LA09042 and MSM0021620859.

${ }^{\text {s }}$ Supported by the Swiss National Science Foundation.

${ }^{\mathrm{t}}$ Supported by CONACYT, México, grant 48778-F.

unussian Foundation for Basic Research (RFBR), Grant No. 1329.2008.2 and Rosatom.

${ }^{\mathrm{v}}$ Supported by the Romanian National Authority for Scientific Research under the contract PN 09370101.

${ }^{w}$ Partially Supported by Ministry of Science of Montenegro, No. 051/3-3352.
} 
a)

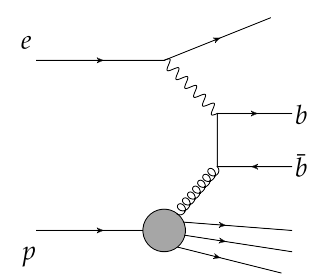

b)

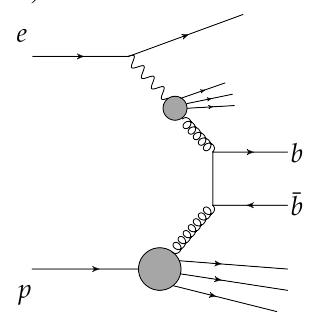

Fig. 1 Generic leading order diagrams for $b \bar{b}$ production in $e p$ collisions. The diagram (a) is referred to as direct or pointlike, the diagram (b) is referred to as resolved or hadronlike

quantum chromodynamics (QCD) due to the large scale provided by the mass of the heavy $b$-quark and the correspondingly small coupling $\alpha_{s}$. Resolved processes where the photon fluctuates into a hadronic state before undergoing a hard collision, as indicated in Fig. 1b, are expected to be largely suppressed compared to the direct production process, because of the large $b$-quark mass. Due to the dominance of the direct process over the resolved process, the production of $b$-quarks in $e p$ collisions at HERA is an excellent testing ground for QCD predictions.

Theory uncertainties in the prediction of the cross section, which are mainly related to the renormalisation and factorisation scales, are expected to be smaller for beauty production than for charm production. The study of beauty photoproduction near threshold is of particular theoretical interest as the only hard scale in this process is provided by the $b$-quark mass, and other scales like the photon virtuality ( $Q^{2} \approx 0 \mathrm{GeV}^{2}$ in photoproduction) or the transverse momentum of the $b$-quark can be neglected.

At HERA the beauty cross section in photoproduction $e p \rightarrow e b \bar{b} X$ has been measured by the H1 [1-5] and ZEUS [6-12] collaborations and compared to calculations [13-15] at next-to-leading order (NLO) QCD, performed in the fixed flavour number scheme in which the beauty quark is treated as massive. In general the predictions using the factorisation and renormalisation scale $\mu_{R}=\mu_{F}=\sqrt{m_{b}^{2}+P_{T}(b)^{2}}$ do not agree well with the data. In particular at low values of the transverse momentum of the beauty quarks $P_{T}(b) \approx 0 \mathrm{GeV}$, i.e. in the phase space region where the only hard scale involved is the $b$-quark mass $m_{b}$, the measurements show a tendency to lie above the prediction. The choice of a lower scale, $\mu_{R}=\mu_{F}=1 / 2 \sqrt{m_{b}^{2}+P_{T}(b)^{2}}$, leads to a better agreement of the prediction with the data [16].

In the present analysis a measurement of the differential beauty cross section at HERA in photoproduction as function of the quadratically averaged transverse momentum of the produced beauty quarks, $\mathrm{d} \sigma / \mathrm{d}\left\langle P_{T}(b)\right\rangle$, is made down to the $b \bar{b}$-production threshold, using a novel technique based on low momentum electron identification.

Most of the previous beauty measurements at HERA in photoproduction and deep-inelastic scattering (DIS, $Q^{2} \gtrsim 1 \mathrm{GeV}^{2}$ ) identified jets of $b$-quarks using single leptons tags $[1,4,5,7,9-11,17-20]$ or displaced vertices [2, 6, 21-24]. Jet-based $b$-tagging algorithms are in general very efficient at high transverse momenta of $b$-quarks, $P_{T}(b)>6-7 \mathrm{GeV}$, but degrade significantly for lower values due to the absence of the boost and the short decay length. In addition $b$-quarks almost at rest lead to isotropic decay topologies of the final state where jet finders usually fail. A second class of analyses used double tags to select $b \bar{b}$ pairs either by reconstructing two muons [8] or a muon and a $D^{*}$ meson $[3,10]$, utilising the semileptonic decay channel $b \rightarrow \mu X^{\prime}$ and the decay channel $b \rightarrow D^{*} X^{\prime}$, respectively. Lower values of the transverse momentum of the $b$-quarks become accessible by the use of lepton tags without requiring jets, where the minimum $P_{T}(b)$ value is determined by the minimum transverse momentum cut on the lepton. For muons this cut is typically at $P_{T}(\mu) \approx 2 \mathrm{GeV}$, and therefore too high to measure efficiently the production cross sections of $b$-quarks near threshold.

In the present analysis the differential beauty cross section is measured using electron pairs, exploiting the doublesemileptonic decay $b \bar{b} \rightarrow e e X^{\prime}$, with online and offline $P_{T}(e)$ thresholds for the electron identification of about $1 \mathrm{GeV}$. The events were recorded by identifying low momentum electrons already online using a dedicated trigger, which recorded data in the year 2007 with a corresponding integrated luminosity of $48.1 \mathrm{pb}^{-1}$. This low cut on the transverse electron momentum, $P_{T}(e)$, improves not only the total acceptance but also makes the low $P_{T}(b)$ phase space experimentally accessible.

\section{Monte Carlo simulations and QCD calculations}

The Monte Carlo generators PYTHIA $[25,26]$ and CASCADE [27-29] are used to determine the signal efficiency and the detector acceptance for the process $e p \rightarrow e b \bar{b} X \rightarrow$ $e e e X^{\prime}$, and to simulate the production of charm quarks. Differences in the predictions are taken into account as systematic uncertainty, see Sect. 5.4. For the production of $J / \psi$ mesons only CASCADE and for the production of light quarks in photoproduction only PYTHIA is used. Deepinelastic scattering is simulated using the Monte Carlo generator RAPGAP [30].

In PYTHIA leading order matrix elements are implemented taking into account the mass of the heavy quarks. The CTEQ6L [31] set of proton parton density functions is used. The parton shower evolution in PYTHIA is based on the DGLAP equations [32-34]. In addition to the direct process, the resolved photon component is calculated by using the photon parton density function SAS 2D [35]. 
For the CASCADE simulation the direct $\gamma^{*} p \rightarrow b \bar{b}$ and $\gamma^{*} p \rightarrow c \bar{c}$ processes are implemented using off-shell matrix elements, which are convoluted with $k_{T}$-unintegrated ${ }^{1}$ proton parton density functions. The A0 [36] set of parton density functions is used. The parton evolution in CASCADE is based on the CCFM evolution equation [37-40] for the initial state parton shower.

In PYTHIA, CASCADE and RAPGAP higher order QCD corrections are included by simulating parton showers in the initial and final state. These Monte Carlo generators use the Lund String Model [41, 42] for simulating the hadronisation of light quarks. For the hadronisation of heavy quarks the Bowler fragmentation model [43] is employed with parameters as used in a previous analysis [21].

In order to correct for detector effects and to estimate the systematic uncertainties associated with the measurement, the generated events are passed through a detailed simulation of the detector response based on the GEANT program [44] and through the same reconstruction and analysis software as is used for the data.

Theory cross sections are calculated in NLO QCD in the fixed flavour number scheme using the program FMNR [13-15] in order to compare with the data. These calculations are expected to give reliable results in the kinematic region considered here, where the transverse momentum of the heavy quark is of the same order of magnitude as its mass. The calculations are performed as a function of the quadratically averaged transverse momentum of the produced beauty pair

$$
\left\langle P_{T}(b)\right\rangle=\sqrt{\left(P_{T, b}^{2}+P_{T, \bar{b}}^{2}\right) / 2} .
$$

The prediction of FMNR is evaluated for the direct and resolved photon processes. For the proton the CTEQ6M [31] set and for the photon the GRV-HO [45] set of parton density functions are used. In this analysis, the renormalisation and factorisation scales are chosen to be equal, $\mu_{R}=\mu_{F}=$ $\mu_{0}$, with $\mu_{0}=1 / 2 \sqrt{m_{b}^{2}+\left\langle P_{T}(b)\right\rangle^{2}}$ and $m_{b}=4.75 \mathrm{GeV}$. The value used for the QCD scale $\Lambda_{\mathrm{QCD}}$ corresponds to the value of the strong coupling constant $\alpha_{s}\left(M_{\mathrm{Z}}\right)=0.118$. The theoretical uncertainty of the prediction is evaluated by varying the scales $\mu_{R}$ and $\mu_{F}$ simultaneously in the window $\mu_{0} / 2<\mu_{R, F}<2 \mu_{0}$ and the beauty mass in the range $4.5<m_{b}<5.0 \mathrm{GeV}$. By recalculating the cross section with different parton density functions the theoretical uncertainty due to the choice of the photon and proton parton density functions is found to be much smaller than the theoretical uncertainties and thus is neglected.

\footnotetext{
${ }^{1} k_{T}$ denotes the transverse momentum of the parton.
}

\section{H1 detector}

A detailed description of the $\mathrm{H} 1$ detector can be found elsewhere [46-48]. In the following, only detector components relevant to this analysis are briefly discussed. The origin of the $\mathrm{H} 1$ coordinate system is the nominal ep interaction point, with the direction of the proton beam defining the positive $z$ axis (forward direction). Transverse momenta are measured in the $x-y$ plane. Polar $(\vartheta)$ and azimuthal $(\phi)$ angles are measured with respect to this reference system. The pseudorapidity is defined to be $\eta=-\ln \tan (\vartheta / 2)$.

In the central region $\left(15^{\circ}<\vartheta<165^{\circ}\right)$ the interaction point is surrounded by the central tracking detector (CTD). The CTD comprises two large cylindrical jet chambers (CJC1 and CJC2) and the silicon vertex detector [49]. The CJCs are separated by a drift chamber which improves the $z$ coordinate reconstruction. The CTD detectors are arranged concentrically around the interaction region in a solenoidal magnetic field of $1.16 \mathrm{~T}$. The trajectories of the charged particles are measured with a transverse momentum resolution of $\sigma\left(p_{T}\right) / p_{T} \approx 0.2 \% p_{T} / \mathrm{GeV} \oplus 1.5 \%$. In addition the CJCs provide a measurement of the specific ionisation energy loss $\mathrm{d} E / \mathrm{d} x$ of charged particles with a relative resolution of $6.5 \%$ for long tracks. A set of five cylindrical multiwire proportional chambers [50] mainly used for first level triggering are situated inside the inner $\mathrm{CJC} 1$ covering the polar angular region $11^{\circ}<\vartheta<169^{\circ}$. The forward tracking detector and the backward proportional chamber measure tracks of charged particles at smaller $\left(7^{\circ}<\vartheta<25^{\circ}\right)$ and larger $\left(155^{\circ}<\vartheta<175^{\circ}\right)$ polar angles than the central tracker, respectively.

The liquid argon (LAr) sampling calorimeter [51] surrounds the tracking chambers and has a polar angle coverage of $4^{\circ}<\vartheta<154^{\circ}$. It consists of an inner electromagnetic section with lead absorbers and an outer hadronic section with steel absorbers. The LAr calorimeter is divided into eight wheels along the beam axis. The electromagnetic and the hadronic sections are highly segmented in the transverse and the longitudinal directions. Energies of electromagnetic showers are measured with a precision of $\sigma(E) / E=12 \% / \sqrt{E / \mathrm{GeV}} \oplus 1 \%$ and energies of hadronic showers with $\sigma(E) / E=50 \% / \sqrt{E / \mathrm{GeV}} \oplus 2 \%$, as determined in test beam experiments [52, 53]. In the backward region $\left(153^{\circ}<\vartheta<178^{\circ}\right)$, particle energies are measured by a lead-scintillating fibre calorimeter (SpaCal) [48].

The luminosity is determined from the rate of the elastic QED Compton process $e p \rightarrow e \gamma p$, with the electron and the photon detected in the SpaCal calorimeter, and the rate of DIS events measured in the SpaCal calorimeter [54].

For data collection a four level trigger system is employed, of which the first two levels are implemented in hardware. The first level trigger (L1) is based on various sub-detector components, which are combined and refined 
at the second level (L2). The third level (L3) is a software based trigger using combined L1 and L2 trigger information from various subdetector components. Fully reconstructed events are subject to an additional selection at the software filter farm (L4).

The data used for this measurement were recorded by the Fast Track Trigger (FTT) [55-60] which, based on hit information provided by the CJCs, reconstructs tracks with subsequently refined granularity at the first two trigger levels, first in the $x-y$ plane at L1 and then in three dimensions at L2. Of special importance is the third trigger level integrated in the FTT [60], which identifies low energy electrons $(E>1 \mathrm{GeV})[61,62]$ by combining FTT tracks with energy depositions reconstructed in the LAr calorimeter by the Jet Trigger (JT) [63].

\section{Experimental method}

The data sample used for this analysis was recorded in the year 2007, when positrons at an energy of $27.6 \mathrm{GeV}$ collided with protons at $920 \mathrm{GeV}$, and when all trigger levels of the FTT and the JT were in operation. The recorded data corresponds to a total integrated luminosity of $48.1 \mathrm{pb}^{-1}$.

In this analysis the measurement of $b \bar{b}$ photoproduction is based on the identification and selection of two electrons in the LAr calorimeter at low transverse momentum $P_{T}(e)>1 \mathrm{GeV}$ to tag the semi-electronic decays of the $b$ quarks. In about $2 \%$ of all $b \bar{b}$-decays two electrons originate from the same $b$-quark from the decay chain $b \rightarrow$ $c e^{-} \bar{v}_{e} \rightarrow s e^{-} e^{+} \bar{v}_{e} v_{e}$. In about $4 \%$ of all $b \bar{b}$-decays the two electrons originate from decays of different $b$-quarks, where they are either produced directly in the semi-electronic $b$ decays or in the subsequent semi-electronic $c$-decays. The electrons can be either of opposite charge (combinations $b \bar{b} \rightarrow e^{+} e^{-} X$ and $c \bar{c} \rightarrow e^{+} e^{-} X$ ) or of same charge (combinations $b \bar{c} \rightarrow e^{-} e^{-} X$ and $\left.\bar{b} c \rightarrow e^{+} e^{+} X\right)$. These charge relations hold only in the case of no $B \bar{B}$ mixing. In the following all possible combinations including $B \bar{B}$ mixing are considered in order to discriminate $b \bar{b}$ decays against semielectronic decays of $c \bar{c}$ events. Electron pairs from $J / \psi$ decays are distinguished from those from $b$-decays by reconstructing their invariant mass. Misidentified electrons originating mainly from the light quark background are con- strained by varying the cuts on the electron identification described in Sect. 5.

\subsection{Online electron identification}

Events containing several tracks and one or two electron candidates compatible with the signature of semi-electronic $b$-decays are triggered, using the FTT on the trigger levels L1 to L3. On the first trigger level more than five tracks with transverse momentum thresholds in the range $0.1-1.8 \mathrm{GeV}$ are required. These high multiplicities are verified at the second trigger level, exploiting the higher track resolution available at this level. On the third trigger level the track information as determined by FTT-L2 is combined with the energy depositions as measured in the LAr calorimeter by the Jet Trigger [60-62] to identify electrons. Electron candidates are required to fulfil a geometrical track-cluster matching condition using the distance variables $\Delta \vartheta=\left|\vartheta_{\mathrm{FTT}}-\vartheta_{\mathrm{JT}}\right|$ and $\Delta \varphi=\left|\varphi_{\mathrm{FTT}}-\varphi_{\mathrm{JT}}\right|$. In addition the transverse momentum $P_{T \text {,FTT }}$ as measured with the FTT-L2 has to be compatible with the associated transverse energy $E_{T, \mathrm{JT}}$ measured in the LAr calorimeter by the JT. A lower cut on the quantity $E_{T, \mathrm{JT}} / P_{T, \mathrm{FTT}}$ is used to discriminate electrons against hadrons, which deposit significantly less energy in the noncompensating LAr calorimeter.

For this analysis three subtriggers are used, which have identical L1 and similar L2 trigger conditions, but different conditions on L3 as summarised in Table 1. The subtrigger with the lowest transverse momentum threshold of $P_{T, \text { FTT }}>1.2 \mathrm{GeV}$ requires events with at least two electron candidates. The other two subtriggers select events with a minimum of one electron candidate with $P_{T \text {,FTT }}$ thresholds of 1.5 and $2.0 \mathrm{GeV}$. The three data sets recorded by these FTT-JT based subtriggers cover an overlapping kinematic phase space, but correspond to different integrated luminosities due to different trigger prescale factors. The three data sets are combined using a weighting method [61] to account for correlated triggers with prescales. The individual prescale corrected luminosities are also given in Table 1.

\subsection{Offline electron identification}

Electrons in the polar angle range of $20^{\circ}<\vartheta(e)<140^{\circ}$ and with a transverse momentum of $P_{T}(e)>1 \mathrm{GeV}$, with

Table 1 L3-online cuts used to trigger electron candidates. Explanations to the cuts are given in the text. The last column contains the prescale corrected integrated luminosity of each subtrigger. The medium- $P_{T}$ subtrigger was commissioned at a later stage

\begin{tabular}{|c|c|c|c|c|c|c|}
\hline Subtrigger & \# ele. cand. & $P_{T, \text { FTT }}[\mathrm{GeV}]$ & $\frac{E_{T, \mathrm{JT}}}{P_{T, \mathrm{FTT}}}[\%]$ & $\Delta \varphi[\mathrm{rad}]$ & $\Delta \vartheta[\mathrm{rad}]$ & $\mathcal{L}\left[\mathrm{pb}^{-1}\right]$ \\
\hline Low- $P_{T}$ & $\geq 2$ & $>1.2$ & $>30$ & $<0.30$ & $<0.25$ & 25.1 \\
\hline Medium- $P_{T}$ & $\geq 1$ & $>1.5$ & $>50$ & $<0.15$ & $<0.20$ & 13.1 \\
\hline High- $P_{T}$ & $\geq 1$ & $>2.0$ & $>60$ & $<0.20$ & $<0.20$ & 33.5 \\
\hline
\end{tabular}


$P_{T}(e)$ and $\vartheta(e)$ measured from the electron track, are identified using energy depositions in the LAr calorimeter and specific ionisation loss measured in the CJCs. Two estimators, $D_{\text {calo }}$ and $D_{\mathrm{d} E / \mathrm{d} x}$, are defined to discriminate electrons from background. The background, which is mainly due to pions misidentified as electrons and to a lesser extent due to kaons and anti-protons, is largely suppressed by combining the two independent estimators into a combined estimator $D_{\text {ele }}$, as explained in Appendix A. The three estimators are defined such that $D=1$ for genuine electrons and $D=0$ for pion background.

The calorimeter based electron identification [61] is track seeded, which means the cluster shape estimators are calculated from energy deposits in LAr calorimeter cells lying within a cylinder of $30 \mathrm{~cm}$ around the extrapolated track trajectory. The cluster energies are corrected for energy losses in the dead material in front of the LAr calorimeter. Electron candidates with energy depositions close to inactive regions between LAr calorimeter modules are rejected. Five estimators are defined: four cluster shape variables and the ratio of the energy deposited in the electromagnetic part of the LAr calorimeter to the momentum of the corresponding track. These estimators together with the logarithm of the total energy and the $z$ position of the cluster, are mapped onto one single estimator $D_{\text {calo }}$ using the artificial neural network Multilayer Perceptron [64].
The measured specific ionisation loss of the track, $\mathrm{d} E / \mathrm{d} x$, is translated into $\chi^{2}$-probabilities of corresponding particle hypotheses $P(\mathrm{~d} E / \mathrm{d} x, e)$ for electrons and $P(\mathrm{~d} E / \mathrm{d} x, \pi)$ for pions, which constitute the main background. From both probabilities the estimator

$$
D_{\mathrm{d} E / \mathrm{d} x}=\frac{P(\mathrm{~d} E / \mathrm{d} x, e)}{P(\mathrm{~d} E / \mathrm{d} x, e)+P(\mathrm{~d} E / \mathrm{d} x, \pi)}
$$

is constructed. The simulation of the specific ionisation was studied in detail in order to describe precisely the measured energy losses [65].

The performance of both discriminator variables is validated using Monte Carlo and data samples of identified electrons and pions in the transverse momentum range of interest, $1<P_{T}(e)<5 \mathrm{GeV}$, selected in decays $J / \psi \rightarrow$ $e^{+} e^{-}$and $K_{s}^{0} \rightarrow \pi^{+} \pi^{-}$, by means of the "tag and probe method" [61].

The simulation describes well the distribution of the discriminators $D_{\text {calo }}$ and $D_{\mathrm{d} E / \mathrm{d} x}$ as measured in data, as can be seen in Fig. 2. The deviations of the simulation from the data at small $D$ values in the electron sample are due to a small remaining pion contamination in the data. Also the combined estimator $D_{\text {ele }}$ is found to be well described by the simulation and shows an excellent separation of the electron signal from the pion background. Isolated electrons are selected for $D_{\text {ele }}>0.825$ with an efficiency of more than $90 \%$ for a pion background rejection of about $99 \%$.
Fig. 2 Normalised discriminator distributions for the separation of electrons and pions as obtained from $J / \psi \rightarrow e^{+} e^{-}$and $K_{s}^{0} \rightarrow \pi^{+} \pi^{-}$decays using the tag and probe method. (a) The track seeded, calorimeter based discriminator $D_{\text {calo }}$, (b) the discriminator $D_{\mathrm{d} E / \mathrm{d} x}$ based on the measurement of the specific energy loss in the CTD and (c) their combination $D_{\text {ele }}$. Data are represented by circles and Monte Carlo simulations by histograms

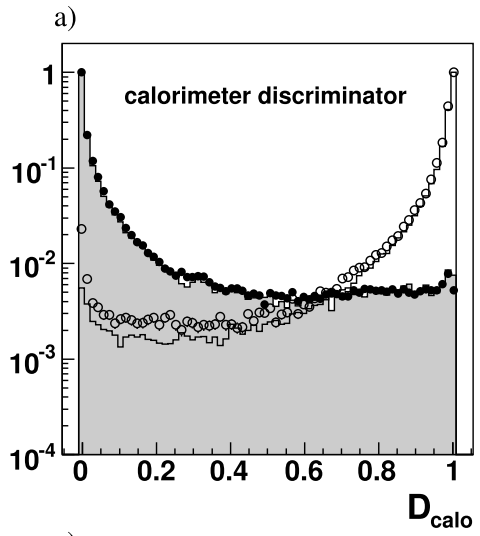

c)
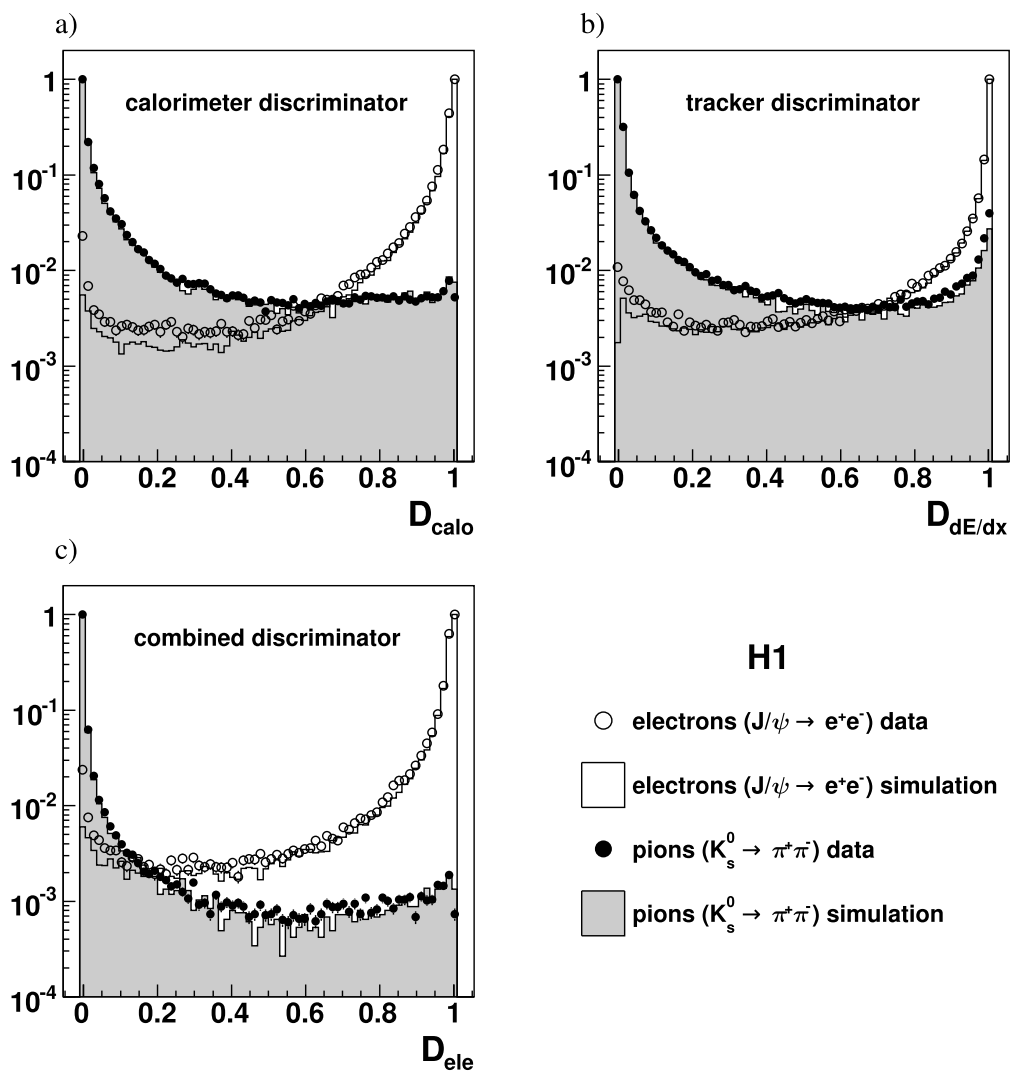

H1

electrons $\left(\mathrm{J} / \psi \rightarrow \mathrm{e}^{+} \mathrm{e}^{-}\right)$data

electrons $\left(\mathrm{J} / \psi \rightarrow \mathrm{e}^{+} \mathrm{e}^{-}\right)$simulation

- pions $\left(\mathrm{K}_{\mathrm{s}}^{0} \rightarrow \pi^{+} \pi^{-}\right)$data

pions $\left(\mathrm{K}_{\mathrm{s}}^{0} \rightarrow \pi^{+} \pi^{-}\right)$simulation 


\subsection{Event selection}

A di-electron sample is obtained by selecting events with two or more offline reconstructed electron candidates, requiring $D_{\text {ele }}>0.825$. To account for the $P_{T}$ resolution of the third trigger level, the $P_{T}$ cut on electron tracks reconstructed offline is raised by $100 \mathrm{MeV}$ above the trigger threshold of the respective subtrigger (see Table 1), which recorded the event. This implies two electrons with $P_{T}>$ $1.3 \mathrm{GeV}$ for the low- $P_{T}$ subtrigger and one electron with $P_{T}>1.6 \mathrm{GeV}\left(P_{T}>2.1 \mathrm{GeV}\right)$ for the medium- $P_{T}$ (high$P_{T}$ ) subtrigger. If the event is triggered by the medium- $P_{T}$ or high- $P_{T}$ subtrigger, the transverse momentum of the second electron has to fulfil $P_{T}>1.0 \mathrm{GeV}$.

In order to remove background from non-ep sources, the measurement of a good event vertex is required. The event vertex is reconstructed from all charged tracks of an event and its position along the beamline has to be within $30 \mathrm{~cm}$ around the nominal interaction point. In addition, timing vetoes are applied to further reduce non- $e p$ interaction induced backgrounds.

In order to reject background from DIS, events with a positron in the LAr calorimeter identified by the standard electron identification [67] and with $E\left(e^{+}\right)>8 \mathrm{GeV}$ are rejected. As the $P_{T}(e)$-distribution of semileptonically decaying $b$-quarks falls steeply, almost all $b$-decay positrons are at low energies and thus not affected by this cut. DIS events with an electromagnetic cluster in the SpaCal calorimeter with an energy above $8 \mathrm{GeV}$ consistent with originating from the scattered beam positron [66] are also rejected. Events with $Q^{2} \lesssim 2 \mathrm{GeV}^{2}$ are not rejected by these cuts, since the beam positrons leave the detector undetected along the beam pipe.

Only events with measured inelasticities in the phase space region of this measurement, $0.05<y_{\mathrm{h}}<0.65$, are accepted. The inelasticity variable is reconstructed from the sum over all final state particles $y_{\mathrm{h}}=\sum_{i}\left(E_{i}-P_{z, i}\right) /$ $\left(2 E_{\mathrm{e}^{+} \text {beam }}\right)$, where $E_{\mathrm{e}^{+} \text {beam }}$ denotes the energy of the beam positron. Particles belonging to the hadronic final state (HFS) are reconstructed using a combination of tracks and calorimeter deposits in an energy flow algorithm that avoids double counting [68-70]. $E_{i}$ and $P_{z, i}$ denote the energies and longitudinal momenta of all final state particles, which correspond to the visible hadronic final state in case of photoproduction, and in case of DIS background also includes the scattered positron. The upper cut on the inelasticity suppresses effectively remaining DIS events.

The beauty signal is further enriched by rejecting electron candidates, which are in a dense hadronic environment. For this purpose the variable $R_{\mathrm{E} \text {,cone }}$ is defined as the ratio of the summed energy of all HFS particles in a cone of $18^{\circ}$ around the electron track direction, $E_{\text {cone }}$, to the electron energy $E_{e}$, which must not exceed an upper threshold:

$R_{\mathrm{E}, \mathrm{cone}}=\frac{E_{\text {cone }}}{E_{e}}<350 \%$.

The effect of this cut is twofold: First, it reduces misidentified electron candidates resulting from overlapping showers in the LAr calorimeter. Second, it enriches electrons from semileptonic beauty decays, which are in general isolated from hadrons due to the large $b$-mass.

Finally, electrons from photon conversions are rejected by the three following cuts. First, the distance of closest approach in the transverse plane, $\mathrm{dca}_{e}$, of the electron tracks to the beam line is restricted to be smaller than $0.2 \mathrm{~cm}$. Second, a photon conversion finder searching for displaced vertices is used to identify electrons originating from the photon conversion process $\gamma \rightarrow e^{+} e^{-}$. Third, the invariant mass of the selected electron pairs is required to be $m_{e 1, e 2}>1.2 \mathrm{GeV}$. This cut rejects $e^{+} e^{-}$pairs from Dalitz decays and most of the remaining background from photon conversions.

The selection cuts are summarised in Table 2. After applying all cuts about 1500 electron pairs are selected. In the rare cases with more than two selected electrons per event all pair combinations are considered in the analysis.

\section{Data analysis}

The selected di-electron sample is dominated by events from inelastic $J / \psi$-meson production. While decays of $J / \psi$ mesons can be easily identified by kinematic reconstruction of the $J / \psi$ mass peak, the separation of the $b \bar{b}$ signal events from the other backgrounds originating from the production of light quarks and open charm production is more difficult.

In the following, the reconstruction of the transverse momentum of the produced $b$-quarks and the flavour separation of the different processes are described.

\subsection{Reconstruction of $b$-quarks}

The transverse momentum of $b$-quarks is reconstructed for the measurement of the differential cross section $\mathrm{d} \sigma / \mathrm{d}\left\langle P_{T}(b)\right\rangle$, where $\left\langle P_{T}(b)\right\rangle$ is the quadratically averaged transverse momentum of the $b$ and $\bar{b}$ quark as defined in (1). The $b \bar{b}$ cross section is largest at small transverse momentum at $\left\langle P_{T}(b)\right\rangle \approx m_{b}$, a kinematic region where standard jet finders cannot be used due to isotropic decay topologies. Therefore an alternative, referenced as the hemisphere method, is exploited. This method was applied in a previous analysis [72] to reconstruct the directions and momenta of charm quarks in the production of $c \bar{c}$-pairs in DIS, and is also well suited to reconstruct the transverse momenta of $b$-quarks in $b \bar{b}$ production [61]. 
Table 2 Overview of the online and offline selection cuts. More details on the selection procedure can be found in [61]
Overview of the selection cuts

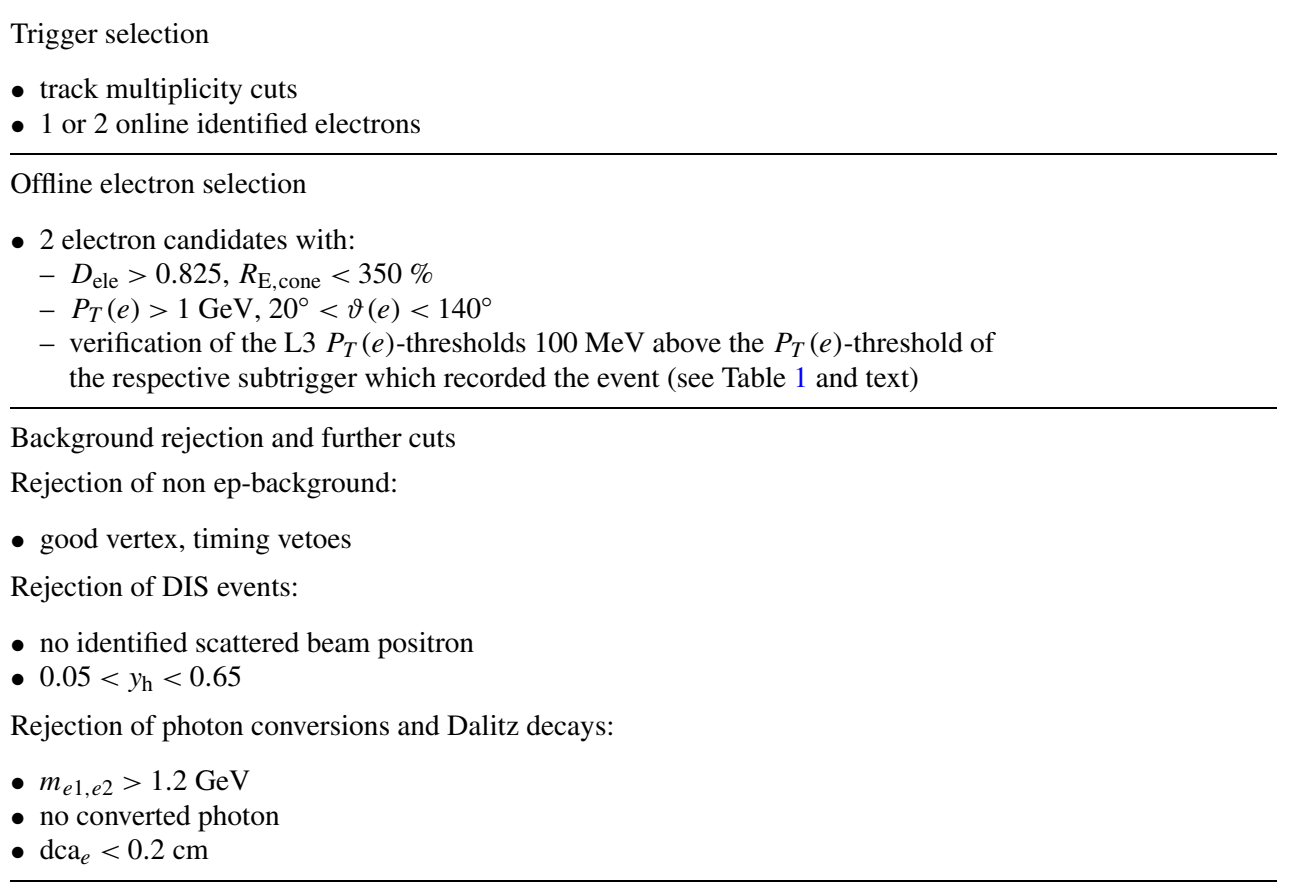

As illustrated in Fig. 3, an event is divided into hemispheres, using the thrust-axis which is calculated in the laboratory frame in the plane transverse to the beam directions ( $x-y$ plane). Using the transverse momenta from all particles of the HFS, the thrust-axis in the transverse plane is given by the vector $\vec{a}$ maximising the sum of the projected transverse momenta onto it,

$T=\max (\vec{a})\left(\frac{\sum_{i \in \mathrm{HFS}}\left|\vec{a} \cdot \vec{P}_{T, i}\right|}{\sum_{i \in \mathrm{HFS}}\left|\vec{P}_{T, i}\right|}\right) \quad$ with $|\vec{a}|=1$.

A plane perpendicular to the thrust-axis defines two hemispheres, one of them containing the fragmentation products of the $b$-quark, and the other one containing the fragmentation products of the $\bar{b}$-quark.

Two observables $\vec{P}_{T \text {, hem.I }}$ and $\vec{P}_{T \text {, hem.II }}$ are used to reconstruct the mean transverse momentum of the $b(\bar{b})$ quark produced in the hard interaction. These observables, which are derived from the HFS particles assigned to the corresponding hemispheres, show a good correlation to the transverse momentum of the $b(\bar{b})$ quarks in the hard process. However, the hadronic final state also contains particles from the so called proton remnant, leaving the interaction in the positive $z$-direction of the detector and thus deteriorating the above correlation. Simulation studies show that the correlation with the $b$-quark transverse momentum is improved by excluding particles in the forward direction at polar angles below 15 degrees. The transverse momenta of the $b(\bar{b})$ -

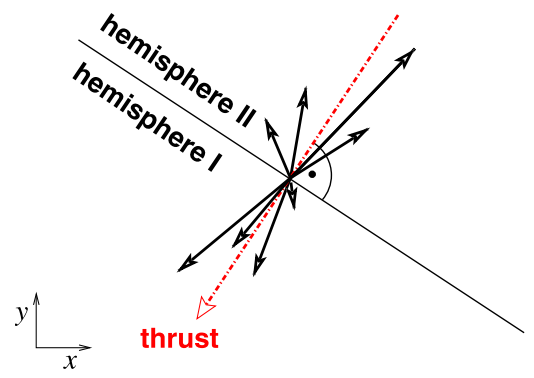

Fig. 3 Schematic illustration of the determination of the thrust axis in the plane transverse to the $e p$ beams. The transverse thrust axis, indicated by the dashed arrow, maximises the sum of momenta projected onto it in this plane. The thrust axis allows the event to be divided into two hemispheres, each containing the decay products of a beauty quark, used to reconstruct the average transverse beauty mass $m_{T, \text { rec }}(b)$ as defined in (7)

quarks are therefore approximated by:

$$
\vec{P}_{T, \text { hem.I (hem.II) }}=\sum_{\substack{i \in \text { hem.I } \\(i \in \text { hem.II })}} \vec{P}_{T, i} \quad \text { with } \vartheta_{i}>15^{\circ}
$$

This reconstruction method is very reliable at large $P_{T, b}$, where two hard jets are measured in the final state. At small $P_{T}(b)$ the transverse momenta of HFS particles in the hemispheres are mainly generated by the $b$ and $\bar{b}$-hadron decays themselves and are related to the mass of the $b$-quark: $\left|\vec{P}_{T, \text { hem.I }}\right| \approx\left|\vec{P}_{T, \text { hem.II }}\right| \approx m_{b}$. In order to allow for a good reconstruction of $P_{T}(b)$ down to the $b \bar{b}$ production threshold, i.e. $P_{T}(b) \approx 0 \mathrm{GeV}$, the average transverse beauty mass 
is used:

$m_{T}(b)=\sqrt{m_{b}^{2}+\left\langle P_{T}(b)\right\rangle^{2}}$.

Detailed studies [61] demonstrated that the average transverse beauty mass can be well reconstructed from the experimental observables $\vec{P}_{T \text {, hem.I }}$ and $\vec{P}_{T \text {, hem.II }}$ using the relation:

$m_{T, \text { rec }}(b)=\alpha *\left(\left|\vec{P}_{T, \text { hem.I }}\right|+\left|\vec{P}_{T, \text { hem.II }}\right|\right) / 2$,

with $\alpha$ being a constant parameter set to $\alpha=1.09$, such that the correlation between generated and reconstructed $m_{T}(b)$ is maximised. This correlation as obtained by simulation is shown in Fig. 4. For values of $m_{b}$ it in the range $4.5<m_{b}<5.0 \mathrm{GeV}$ the dependence of this correlation on $m_{b}$ is negligible.

\subsection{Quark flavour separation}

For the discrimination of the $b \bar{b}$ signal against remaining background from misidentified electrons and for the separation of the different quark-flavour components contributing to the di-electron signature, a template method is used. Several independent phase space regions are defined such that individual background sources are enhanced in certain regions of the phase space and can be tested while other contributions are suppressed. Finally the $b$-signal ("beauty") and the background contributions are obtained by an unfolding procedure. Background sources determined by this method are the production of light quarks ("uds"), open charm production ("charm") and the production of $J / \psi$ mesons (" $J / \psi$ "). The uds background contains also a small fraction of charm and beauty events, where at least one electron candidate does not originate from a semi-electronic heavy quark decay.

\subsubsection{Fraction of light quarks}

In order to determine the background contributions due to misidentified electrons the data sample is grouped in four regions $B 1, B 2, B 3$ and $S$ using different electron quality criteria on $D_{\mathrm{ele}}^{\min (e 1, e 2)}$ and $R_{\mathrm{E}, \mathrm{cone}}^{\max (e 1, e 2)}$, see Table 3. $D_{\mathrm{ele}}^{\min (e 1, e 2)}$ and $R_{\mathrm{E}, \mathrm{cone}}^{\max (e 1, e 2)}$ are the minimum and maximum value of $D_{\text {ele }}$ and $R_{\mathrm{E} \text {, cone }}$ respectively, of the two electron candidates, which form the electron pair. $B 1, B 2$ and $B 3$ are background enhanced regions and $S$ denotes the electron signal enhanced region, which is defined by tight electron identification and isolation cuts. Templates for the determination of the background fractions are obtained from Monte Carlo simulations, see Fig. 5. More than $70 \%$ of the beauty, charm and $J / \psi$ events populate the signal enhanced bin $S$, since these events contain genuine electrons. The uds events are enriched in the three background bins $B 1, B 2$ and $B 3$, due to misidentified electrons. The measured number of events in

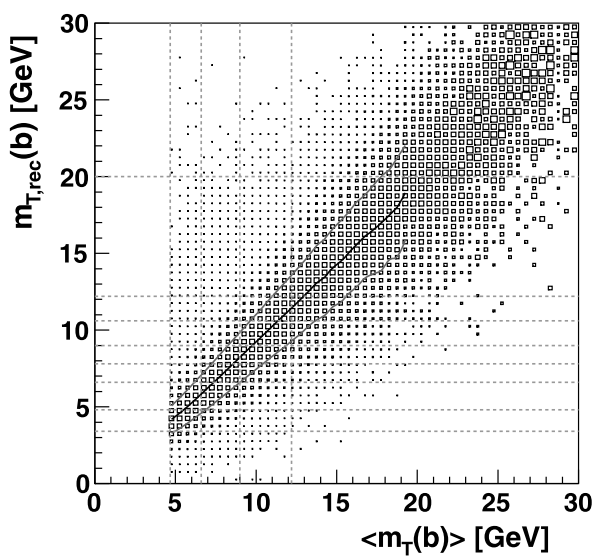

Fig. 4 Correlation between the reconstructed transverse beauty mass $m_{T, \text { rec }}(b)$ and the transverse mass $\left\langle m_{T}(b)\right\rangle$ calculated from the quadratically averaged transverse momentum of the generated beauty quarks. The inner line on the diagonal indicates the correlation of $m_{T, \text { rec }}(b)$ and $\left\langle m_{T}(b)\right\rangle$, and the outer two lines show the $1 \sigma$ error band. The used binning (dotted grey lines) for the vectors $\mathbf{x}$ and $\mathbf{y}$ entering the unfolding procedure are also shown

Table 3 Definition of the four regions used to constrain mainly the uds background

\begin{tabular}{|c|c|c|}
\hline \multirow[t]{2}{*}{$R_{\mathrm{E}, \mathrm{cone}}^{\max (e 1, e 2)}$} & \multicolumn{2}{|l|}{$D_{\text {ele }}^{\min (e 1, e 2)}$} \\
\hline & $0.825-0.875$ & $0.875-1.0$ \\
\hline $150-350 \%$ & $B 1$ & $B 3$ \\
\hline $0-150 \%$ & $B 2$ & $S$ \\
\hline
\end{tabular}

these three background bins mainly constrain the uds background fraction.

\subsubsection{Heavy quark fractions}

In the signal enhanced region $S$, the individual contributions from beauty, charm and $J / \psi$ can be disentangled by investigating the charge product, $q_{e 1} \cdot q_{e 2}$, of the $e^{ \pm}$-candidates, their azimuthal separation $\Delta \phi_{e 1, e 2}=\left|\phi_{e 1}-\phi_{e 2}\right|$, and their invariant mass $m_{e 1, e 2}$. Templates of the different background sources and of the beauty signal, which are all restricted to the signal enhanced region $S$, are shown in Fig. 6 as function of the invariant mass $m_{e 1, e 2}$ and the signed azimuthal separation $\Delta \phi_{e 1, e 2} \cdot q_{e 1} \cdot q_{e 2}$.

The different templates show specific characteristics: $J / \psi$ events have oppositely charged electrons and cluster at $m_{e 1, e 2}=m_{J / \psi}$, whereas background from open charm production covers a large mass range. Electrons from open charm decays are found mostly back-to-back and with opposite charge sign, whereas electron pairs from beauty decays populate all $\Delta \phi_{e 1, e 2}$ values with both charge sign combinations. Both charge products are also found in the uds background, which however populate on average regions with smaller $m_{e 1, e 2}$ values. Large values of $m_{e 1, e 2}$ are solely populated by beauty decays. 
Fig. 5 Templates used to separate the light quarks (uds) from the heavy quark flavours as obtained by the Monte Carlo simulation. For the definition of the background enhanced regions $B 1-B 3$ and the signal enhanced region $S$ see Table 3 and text
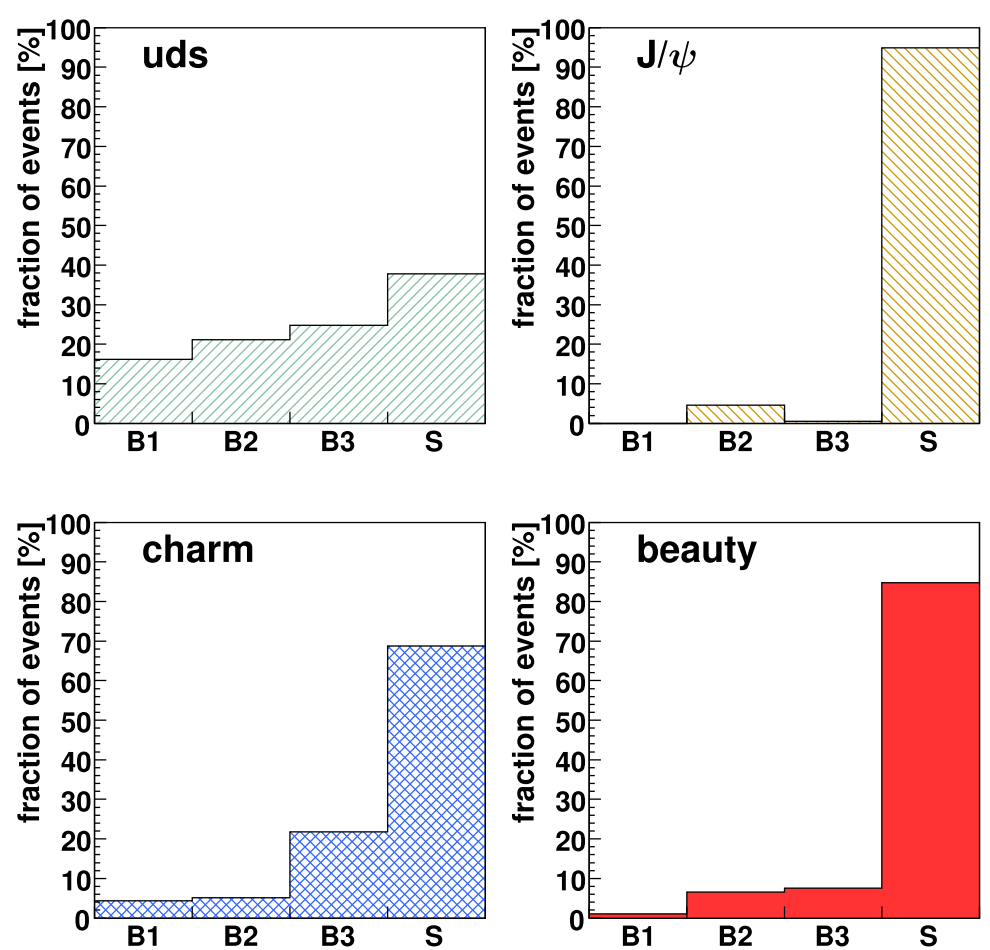

These distinct signatures of the individual background sources, i.e. uds, $J / \psi \rightarrow e e$ and $c \bar{c} \rightarrow e e$, are exploited by dividing the signal enhanced region $S$ into 12 subregions ( $S 1$ to $S 12$ ) as shown in Fig. 6. In the following the three background enhanced bins $B 1-B 3$ and the 12 signal enhanced bins $S 1-S 12$ are referred to as "Flavour Separator", for which templates are derived.

\subsection{Unfolding}

Using an unfolding procedure the number of background events $N_{\mathrm{uds}}, N_{J / \psi}, N_{\text {charm }}$ and the number of beauty events $N_{\text {beauty }, i}$ in four bins of $\left\langle P_{T}(b)\right\rangle$ are derived. A regularised unfolding procedure is used with a smoothness condition. The procedure is explained in Appendix B. All efficiency corrections and migration effects are described by the response matrix A, which correlates the number of reconstructed events in the Flavour Separator distribution in bins of $m_{T \text {,rec }}$, represented by the vector $\mathbf{y}$, with the distribution $\mathbf{x}$ on parton level via the matrix equation

$\mathbf{y}=\mathbf{A x}+\mathbf{b}$.

The vector $\mathbf{x}$, defined as $\mathbf{x}^{T}=\left(\mathbf{x}_{\text {beauty }}^{T}, x_{\text {charm }}, x_{J / \psi}, x_{\mathrm{uds}}\right)$, contains contributions from beauty binned in $\left\langle P_{T}(b)\right\rangle$, charm, $J / \psi$ and uds. The contribution from beauty ( $\mathbf{x}_{\text {beauty }}$ ) is defined according to the phase space given in Table 4 . The vector $\mathbf{b}$ contains the background contribution from DIS events, which is taken from simulation. All other background contributions are incorporated in the response matrix and are determined by unfolding.
Table 4 Definition of the kinematic range of this measurement

\begin{tabular}{l} 
Phase space \\
\hline$Q^{2} \leq 1 \mathrm{GeV}^{2}$ \\
$0.05 \leq y \leq 0.65$ \\
$|\eta(b)|,|\eta(\bar{b})| \leq 2$ \\
\hline
\end{tabular}

Signal and background templates as function of nine $m_{T, \text { rec }}$ bins are generated by Monte Carlo simulations and fitted to the data. The unfolding procedure uses in total $N_{m_{T, \text { rec }}} \times N_{\text {Flavour Separator }}=9 \times 15$ input bins and determines the three background fractions and the number of

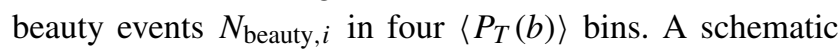
representation of the procedure is shown in Fig. 7. In this procedure the $m_{T, \text { rec }}$ dependences of the different background contributions from uds, $J / \psi \rightarrow e e$ and $c \bar{c} \rightarrow e e$ are fixed by the Monte Carlo predictions. The latter is motivated by recent measurements of the differential cross sections of charm production at HERA, which were found to be consistent with theoretical models and Monte Carlo programs used in this analysis [73, 74].

The fitted beauty signal and background contributions are shown in Fig. 8 in the three background and in the signal enhanced regions. The event numbers resulting from the fitted fractions show very good agreement with the data considering statistical errors only. A clear enhancement of the genuine electron signal due to the tightening of the electron identification cuts is seen when going from the first 
Fig. 6 Templates in the plane $\Delta \phi_{e 1, e 2} \cdot q_{e 1} \cdot q_{e 2}, m_{e 1, e 2}$ and restricted to the signal enhanced region $S$ used to separate the heavy quark flavours as obtained by the Monte Carlo simulation. Also shown is the bin numbering $S 1-S 12$ of the 12 subregions of $S$. For the definition of the signal enhanced region $S$ see Table 3 and text. The two vertical lines indicate the peak invariant mass region of the $J / \psi \rightarrow e^{+} e^{-}$decays
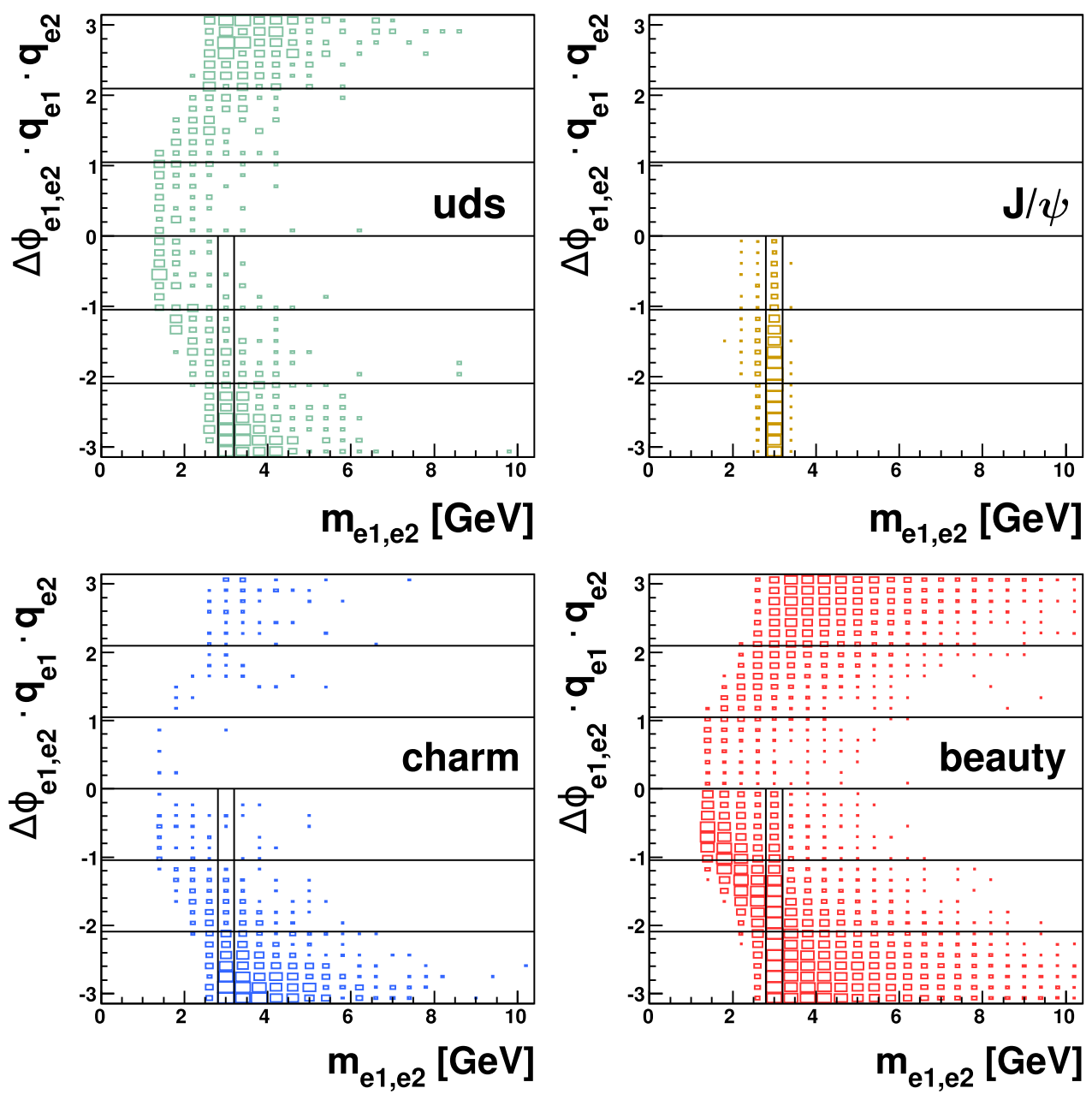

bin numbering

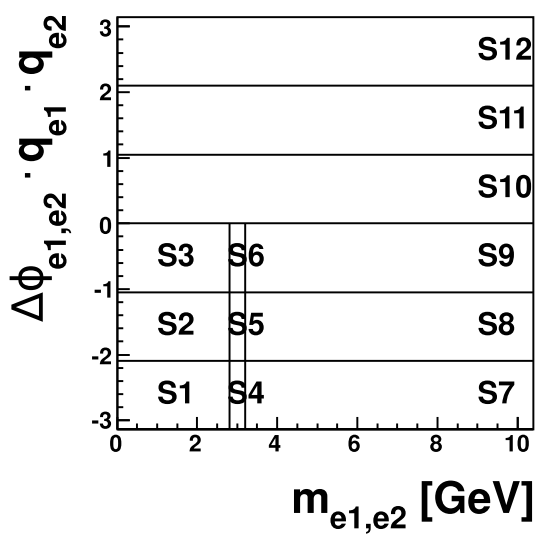

background enhanced region $(B 1)$, which contains more than $80 \%$ uds background to the signal enhanced region ( $S 1-S 12)$ with less than $20 \%$ of uds background.

The correlations between the beauty signal and the background sources, which are largest between beauty and charm, are given in Table 5 together with the determined fractions of the selected data sample.
The distribution of the data as a function of the Flavour Separator is shown in Fig. 9 together with the result from the fit of the beauty and the various background contributions. Good agreement is found considering statistical errors only.

Control distributions of electron variables are presented in Fig. 10 for the electron enriched signal region $(S 1-S 12)$. The data are compared to the simulated beauty signal and 
Fig. 7 Structure of the response matrix $\mathbf{A}$ used to solve the matrix equation $\mathbf{y}=\mathbf{A} \cdot \mathbf{x}+\mathbf{b}$ by unfolding. The indicated numbers specify the number of used bins. The sub-binning in $\mathbf{y}$ given by the Flavour Separator allows the discrimination of the beauty signal from the uds, charm and $J / \psi$ backgrounds. See text for details

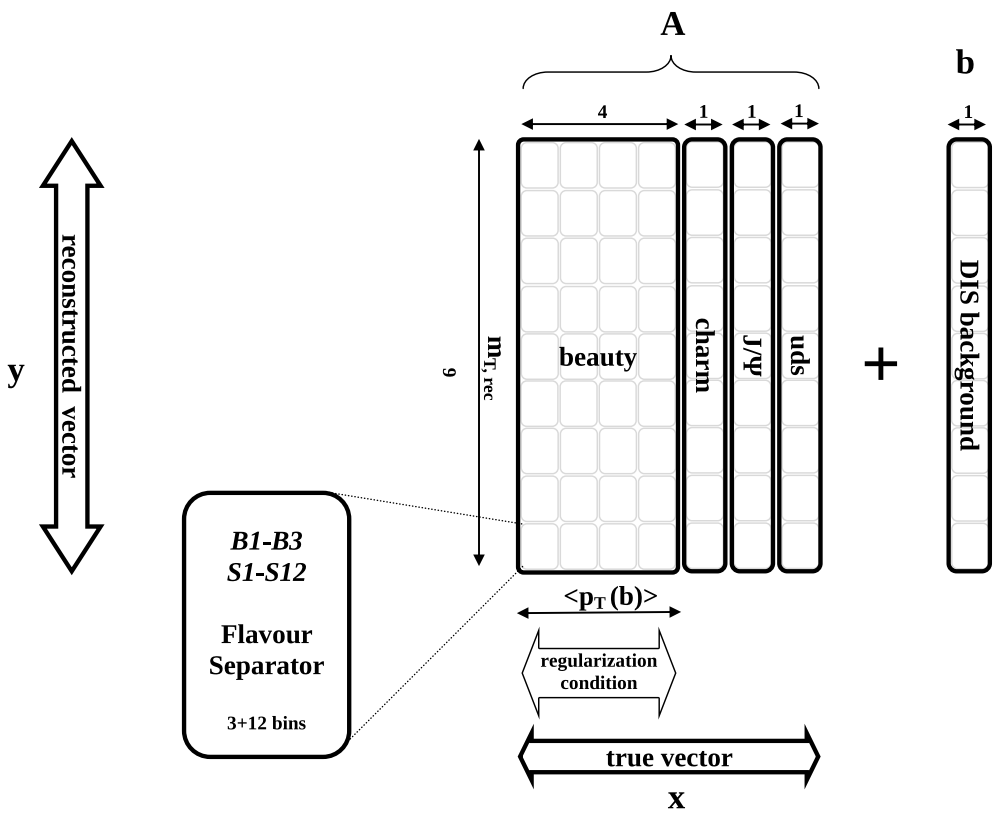

Table 5 Correlations between the signal (beauty) and the different background contributions, and the determined relative fractions with their errors for the data sample. The fraction of DIS events (not given in the table) is $2.3 \%$

\begin{tabular}{llllll}
\hline \multicolumn{2}{l}{ Background correlations } & & & $\begin{array}{l}\text { Fractions } \\
{[\%]}\end{array}$ \\
\cline { 1 - 4 } & beauty & charm & $J / \psi$ & uds & \\
\hline beauty & 1 & -0.46 & -0.18 & -0.18 & $25.8 \pm 3.6$ \\
charm & 1 & -0.03 & -0.27 & $17.6 \pm 3.3$ \\
$J / \psi$ & & 1 & 0.03 & $29.0 \pm 2.1$ \\
uds & & & 1 & $25.3 \pm 3.0$ \\
\hline
\end{tabular}

background distributions using the quark flavour decomposition determined by the unfolding procedure. The main characteristics of the signed variables $\Delta \phi_{e 1, e 2} \cdot q_{e 1} \cdot q_{e 2}$ and $m_{e 1, e 2} \cdot q_{e 1} \cdot q_{e 2}$, and $P_{T}(e)$ and $\vartheta(e)$ are well described by the Monte Carlo simulation. In Fig. 11 additional control distributions are presented showing the $P_{T}$-spectra of the three highest $P_{T}$ tracks. These distributions are strongly dependent on the track trigger conditions used, and imperfections of the trigger simulation would be visible here.

Reasonable agreement between the data and the Monte Carlo simulation is obtained in all distributions which gives confidence that the Monte Carlo simulation is able to correctly model the detector response used for the unfolding procedure.

\subsection{Cross section determination and systematic uncertainties}

The visible cross section is measured for the phase space as defined in Table 4. The bin-averaged differential cross section is obtained as

$$
\frac{\mathrm{d} \sigma(e p \rightarrow e b \bar{b} X)}{\mathrm{d}\left\langle P_{T}(b)\right\rangle}=\frac{N_{\text {beauty }, i}}{\mathcal{L} \cdot B R \cdot \Delta\left\langle P_{T, i}(b)\right\rangle},
$$

where $\mathcal{L}$ is the luminosity, $\Delta\left\langle P_{T, i}(b)\right\rangle$ the bin width, $N_{\text {beauty, } i}$ the number of unfolded signal events in the corresponding bin and $B R=6.17 \%$ the effective branching fraction computed from [71] for a $b \bar{b}$ pair decaying into at least two electrons. For the calculation of cross section uncertainties correlations between bins are taken into account.

The systematic uncertainties related to the measurement of the number of $b \bar{b}$ signal events are listed in the following. The effect on $N_{\text {beauty,i }}$ is calculated by varying the sources of uncertainties in the simulation and by propagating these variations to the measurement through the response matrix A and the background term $\mathbf{b}$ in (8).

- The uncertainty on the electron identification is determined using $J / \psi \rightarrow e^{+} e^{-}$events (see Fig. 2), by comparing the distributions of the electron discriminator $D_{\text {ele }}$ between data and Monte Carlo around each of the used cut values of $D_{\text {ele }}=0.875$ and $D_{\text {ele }}=0.825$. The cut on $D_{\text {ele }}$ is varied in MC by \pm 0.025 which covers any possi-

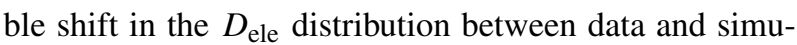
lation. This cut variation on $D_{\text {ele }}$ propagated to the total beauty cross section results in an uncertainty of $\pm 6.8 \%$.

- The uncertainty on the track finding efficiency of electrons is conservatively estimated to be $2 \%$ per track resulting in an uncertainty of the total beauty cross section of $\pm 4 \%$.

- The trigger uncertainty of the FTT at levels L1 and L2 are about $1-2 \%$ each. The dominating contribution to the 


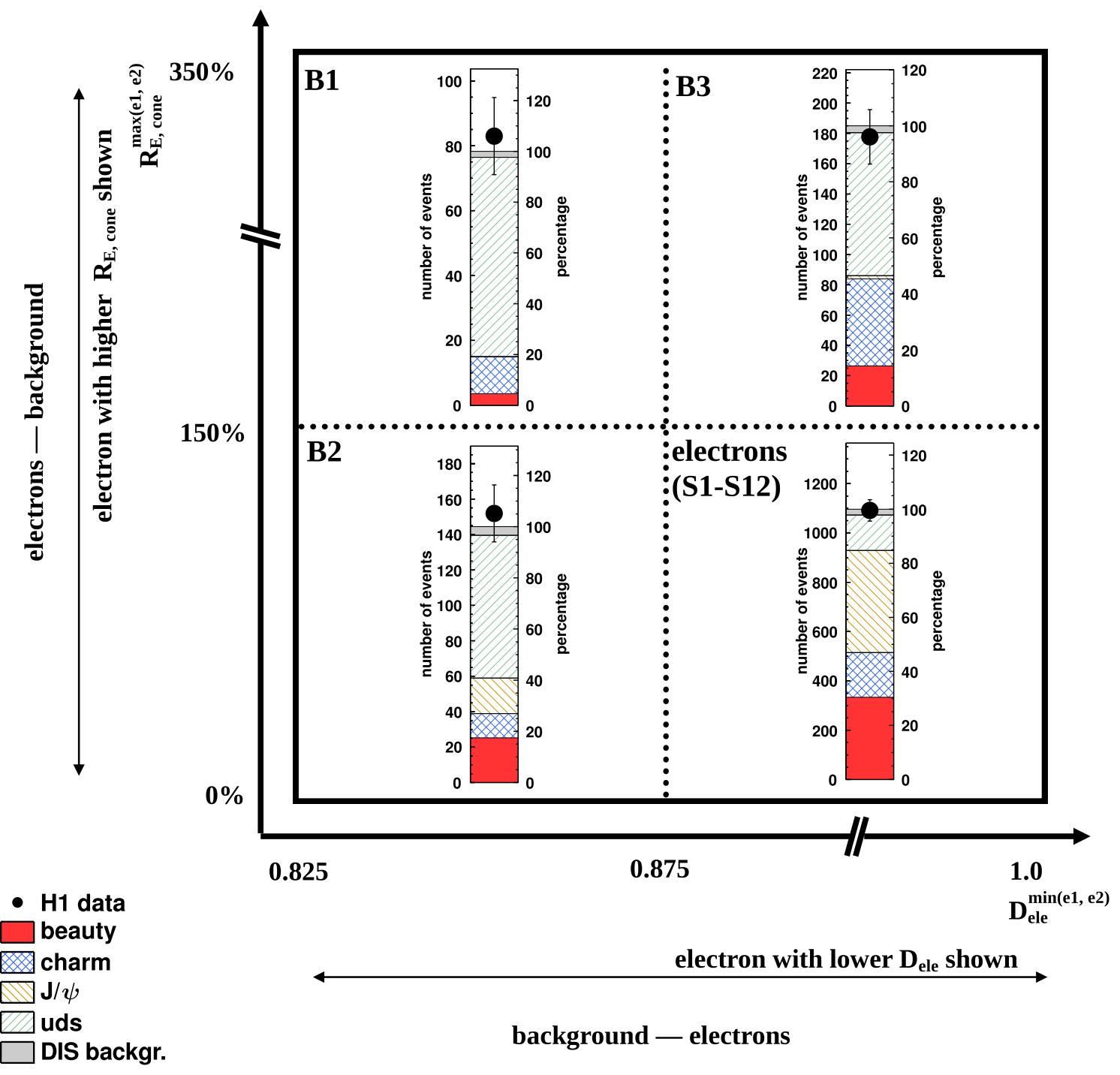

Fig. 8 Number of di-electron events in the background and signal enhanced regions as defined in Table 3. Data are represented as points with the statistical error indicated by the error bars. Also shown in

colour is the decompositions of the event yields as determined by the unfolding procedure

trigger uncertainty is due to the uncertainty of the calibration constants of the JT used at L3. To quantify this uncertainty, the JT calibration constants used in the simulation are varied by scaling the default calibration constants by $15 \%$ [61]. The systematic error on the total beauty cross section due to the uncertainty on the trigger efficiency is determined to be $\pm 8.6 \%$.

- Model uncertainties of the beauty signal are determined by comparing the default response matrix computed by taking the average of the two Monte Carlo samples (CASCADE and PYTHIA) with two alternative response matrices based on one of the Monte Carlo samples. The relative maximum difference with respect to the default response matrix is computed for each entry of the matrix and prop-

agated to a model uncertainty on the total beauty cross section of $\pm 3.3 \%$.

- The uncertainty of the charm contribution is evaluated from the relative difference between the Monte Carlo generators CASCADE and PYTHIA in a similar way as for the beauty signal. The systematic error on the extracted total beauty cross section due to the charm model is determined to be $\pm 3.6 \%$.

- The uncertainty due to the fragmentation function of the heavy quarks is estimated by reweighting the events according to the longitudinal string momentum fraction $z$ carried by the heavy hadron in the Lund model using weights of $(1 \mp 0.7) \cdot(1-z)+z \cdot(1 \pm 0.7)$ for charm quarks and by $(1 \mp 0.5) \cdot(1-z)+z \cdot(1 \pm 0.5)$ for beauty quarks [22]. The corresponding systematic error on the 


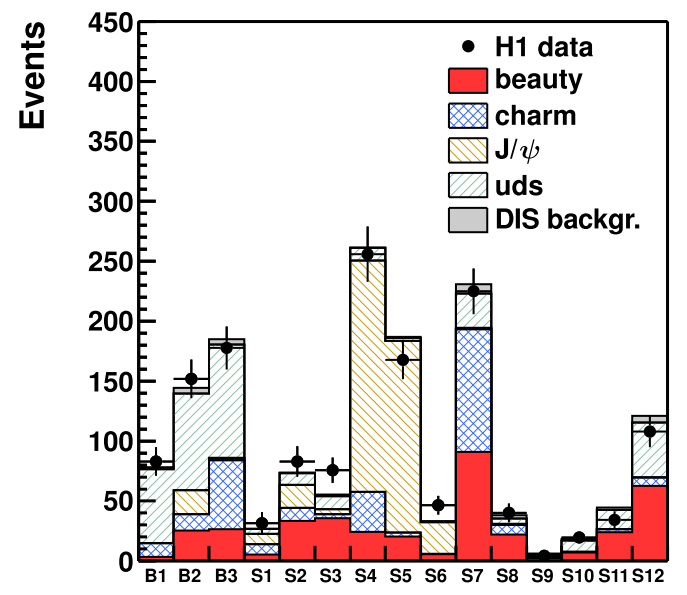

Flavour Separator

Fig. 9 Number of di-electron events in the flavour separator histogram compared to the number of fitted events and their decomposition. Data are represented as points with the statistical uncertainties indicated by the error bars. The bin numbering scheme as defined in Fig. 6 and Table 3 is used total beauty cross section is determined to be $\pm 3.4 \%$ resulting from the charm and $\pm 2.2 \%$ from the beauty fragmentation uncertainty.

- The uncertainty on the contribution from the remaining uds background due to misidentified and real electrons, was determined by varying their relative contributions by a factor two up and down. The corresponding systematic error on the total beauty cross section is determined to be $\pm 3.4 \%$.

- CASCADE does not fully simulate the radiative tail of $J / \psi \rightarrow e e$ events. To estimate the uncertainty on the modelling of it, weights are applied, which are obtained from an elastic $J / \psi \rightarrow e e$ simulation with radiative QED corrections [75]. The systematic uncertainty on the total beauty cross section is estimated to be $\pm 3.5 \%$.

- The uncertainty of the DIS-background, represented in (8) by the vector $\mathbf{b}$, is taken to be $100 \%$ and results in an error on the total beauty cross section of $\pm 4.5 \%$.

In addition, a global normalisation uncertainty of $4.1 \%$ is included with contributions from the integrated luminosity
Fig. 10 Control distributions of the electron candidates compared to Monte Carlo simulations using the quark flavour decomposition determined by the unfolding procedure: (a) signed azimuthal separation $\Delta \phi_{e 1, e 2} \cdot q_{e 1} \cdot q_{e 2}$ defined by the charges multiplied with the azimuthal angle difference of the two electron candidates, (b) signed invariant mass $m_{e 1, e 2} \cdot q_{e 1} \cdot q_{e 2}$ defined by the charges multiplied with the invariant mass of the two electron candidates, (c) polar angle of the electron candidates and (d) transverse momentum of the electron candidates. Data are represented as points with the statistical uncertainties indicated by the error bars. The distributions are restricted to the electron enriched region $(S)$ a)

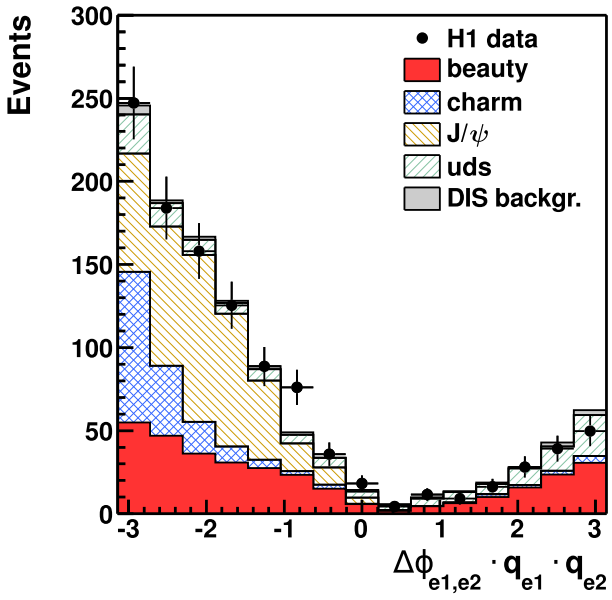

c)

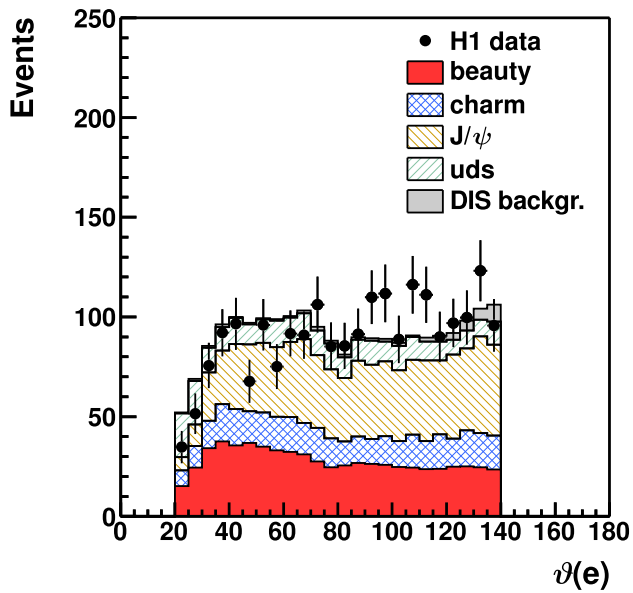

b)

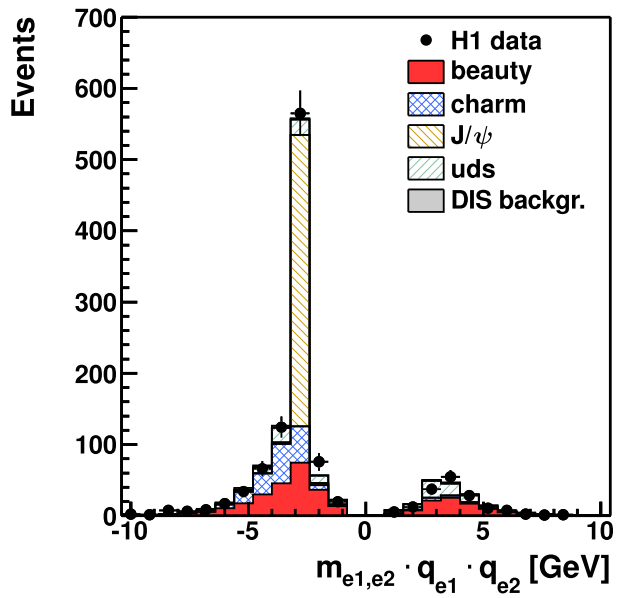

d)

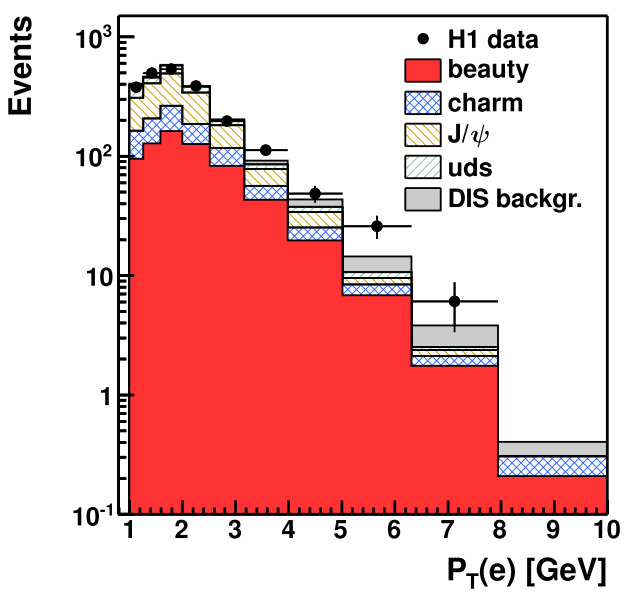


Fig. 11 Control distributions of the hadronic final state as function of the track $P_{T}$. Data are compared to the Monte Carlo simulations using the quark flavour decomposition determined by the unfolding procedure. Data are represented as points with the statistical uncertainties indicated by the error bars for the three highest $P_{T}$ tracks a)

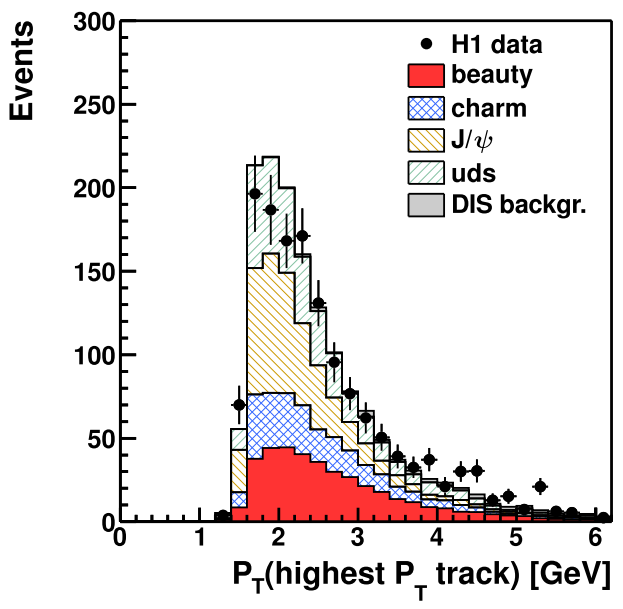

b)

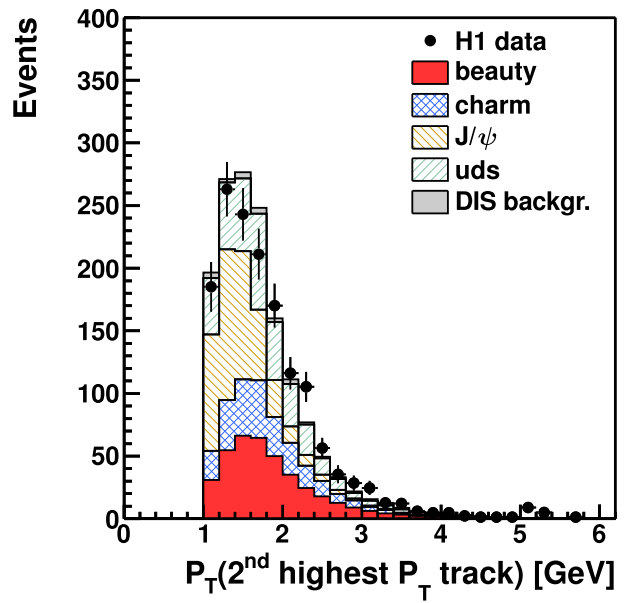

c)

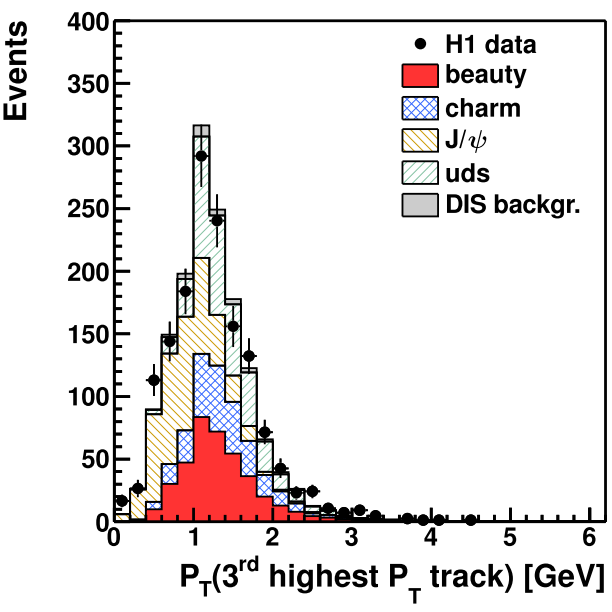

uncertainty of $2.7 \%$ and from the uncertainty on the semielectronic branching fractions of $3.0 \%$.

Adding all above contributions in quadrature gives a total systematic error of $15.4 \%$ on the total beauty cross section.

\section{Results}

The differential cross section $\mathrm{d} \sigma(e p \rightarrow e b \bar{b} X) / \mathrm{d}\left\langle P_{T}(b)\right\rangle$ is measured in the phase space defined in Table 4 using the unfolding procedure as described in Sect. 5.3.

The result is shown in Table 6 together with statistical and total errors and the coefficients describing the statistical correlations between bins. In order to cross check the unfolding procedure the cross section extraction is repeated without regularisation condition. The results obtained with and without regularisation are found to be consistent within the uncertainties.

The measured differential beauty cross section is compared in Fig. 12 with an NLO QCD prediction in the fixed flavour number scheme as calculated by the program FMNR. The figure also shows the ratio of the measured cross section and the NLO QCD cross section. The uncertainties of the measurement are smallest at low $\left\langle P_{T}(b)\right\rangle$, where the cross section is largest. The theoretical prediction of the differential cross section agrees with the measurement within the large experimental and theoretical uncertainties. The prediction has a tendency to be below the data, a trend also observed in previous beauty cross section measurements at large transverse beauty momenta.

By integrating the differential cross section the total inclusive beauty photoproduction cross section is measured as:

$\sigma(e p \rightarrow e b \bar{b} X)=3.79 \pm 0.53$ (stat.) \pm 0.58 (sys.) nb,

to be compared with the NLO prediction obtained from FMNR of $\sigma(e p \rightarrow e b \bar{b} X)=2.40_{-0.49}^{+0.55} \mathrm{nb}$. The measured cross section is higher, but within the large experimental and theoretical uncertainty consistent with the NLO expectation. 


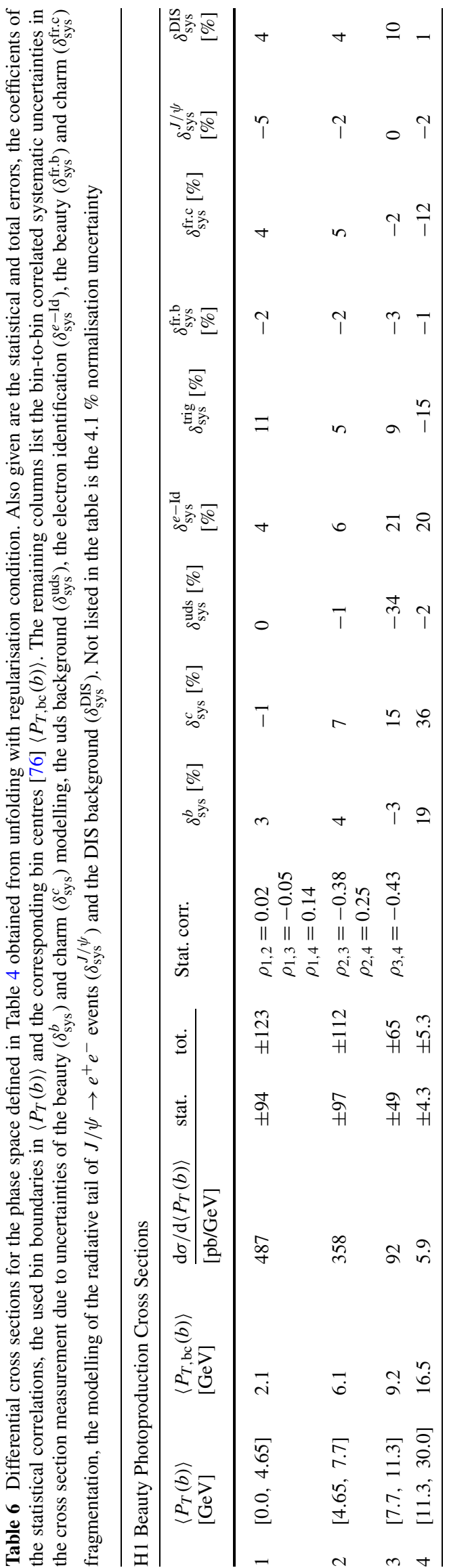

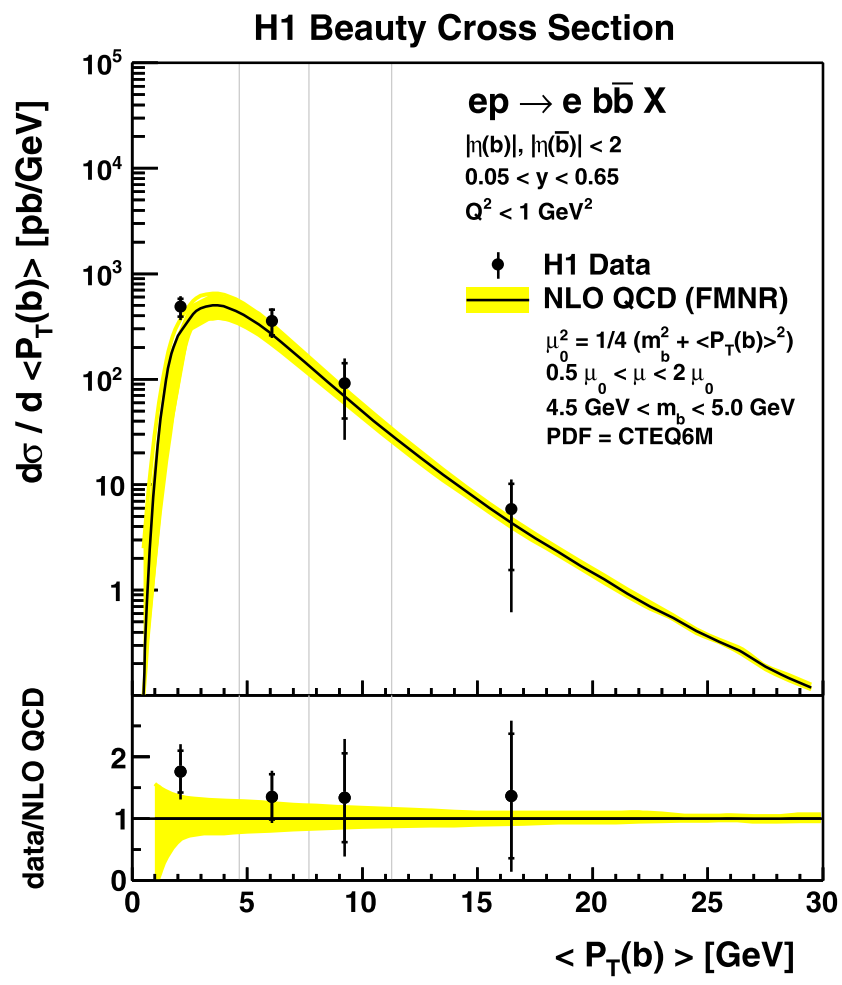

Fig. 12 Differential beauty cross section $\mathrm{d} \sigma / \mathrm{d}\left\langle P_{T}(b)\right\rangle$ shown as function of the quadratically averaged transverse momentum of the beauty quarks $\left\langle P_{T}(b)\right\rangle$ (upper part). The data are represented by points with inner vertical error bars representing the statistical errors and outer error bars representing the total error. The vertical gray lines indicate the bin boundaries in $\left\langle P_{T}(b)\right\rangle$ of each data point and the points are shown at the bin centred positions. The data are compared to the FMNR NLO QCD calculation (solid line) with the uncertainty represented as shaded band. Also shown is the ratio of the measured cross section to the calculated NLO QCD prediction, $\frac{\mathrm{d} \sigma_{\text {measured }}}{\mathrm{d}\left\langle P_{T}(b)\right\rangle} / \frac{\mathrm{d} \sigma_{\mathrm{NLO}} \text { QCD }}{\mathrm{d}\left\langle P_{T}(b)\right\rangle}$ (lower part)

\section{Conclusions}

The inclusive and differential cross section of beauty photoproduction was measured in the di-electron final state, using the $\mathrm{H} 1$ detector at the HERA collider. The cross section is measured as function of the quadratically averaged transverse momentum of the produced beauty quarks $\left\langle P_{T}(b)\right\rangle$, with a special focus on the low $\left\langle P_{T}(b)\right\rangle$ regime. Background from uds, charm and $J / \psi$ production is determined exploiting angular, charge and mass correlations of electron pairs in an unfolding procedure.

The measured cross section is compared to a QCD prediction at NLO performed in the fixed flavour number scheme and evaluated with $\mu_{R}=\mu_{F}=$ $1 / 2 \sqrt{m_{b}^{2}+\left\langle P_{T}(b)\right\rangle^{2}}$ as choice for the renormalisation and factorisation scale. The NLO prediction lies below the data but within the large experimental and theoretical uncertainty they agree.

This measurement is in good agreement with previous measurements of beauty photoproduction at HERA and 
it extends the previously experimentally accessible phase space towards the beauty production threshold.

Acknowledgements We are grateful to the HERA machine group whose outstanding efforts have made this experiment possible. We thank the engineers and technicians for their work in constructing and maintaining the $\mathrm{H} 1$ detector, our funding agencies for financial support, the DESY technical staff for continual assistance and the DESY directorate for support and for the hospitality which they extend to the non-DESY members of the collaboration.

Open Access This article is distributed under the terms of the Creative Commons Attribution License which permits any use, distribu- tion, and reproduction in any medium, provided the original author(s) and the source are credited.

\section{Appendix A: Electron discriminator combination}

The track seeded and calorimeter based electron discriminator $D_{\text {calo }}$ and the electron discriminator $D_{\mathrm{d} E / \mathrm{d} x}$, based on the specific energy loss measurement in the CTD, are mapped to a combined discriminator using the expression

$D_{\text {ele }}(a, b, c, d)=\frac{\left|\left(1-D_{\text {calo }}\right)^{a}-1\right|^{c} \cdot\left|\left(1-D_{\mathrm{d} E / \mathrm{d} x}\right)^{b}-1\right|^{d}}{\left(\left(1-D_{\text {calo }}\right)^{a}-1\right) \cdot\left(\left(1-D_{\mathrm{d} E / \mathrm{d} x}\right)^{b}-1\right)+\left(1-D_{\text {calo }}\right)^{a} \cdot\left(1-D_{\mathrm{d} E / \mathrm{d} x}\right)^{b}}$,

which for the parameter choice $a=b=c=d=1$ corresponds to Bayes' theorem:

$$
\begin{aligned}
& D_{\text {ele }}(1,1,1,1) \\
& \quad=\frac{\left|D_{\text {calo }}\right| \cdot\left|D_{\mathrm{d} E / \mathrm{d} x}\right|}{D_{\text {calo }} \cdot D_{\mathrm{d} E / \mathrm{d} x}+\left(1-D_{\text {calo }}\right) \cdot\left(1-D_{\mathrm{d} E / \mathrm{d} x}\right)} .
\end{aligned}
$$

However, in order to obtain a sensible mapping behaviour of $D_{\text {calo }}$ and $D_{\mathrm{d} E / \mathrm{d} x}$ onto $D_{\text {ele }}$ when their respective values are close to 1 and 0 or both of them are close to 1, the parameters $a=b=0.6$ and $c=d=1.05$ are chosen.

\section{Appendix B: Unfolding procedure}

The differential cross section of beauty photoproduction is extracted from the measured di-electron spectrum using an unfolding procedure as implemented in TUnfold [77].

The vector $\mathbf{y}$, representing the number of measured events, is related via the matrix equation $\mathbf{y}=\mathbf{A} \cdot \mathbf{x}+\mathbf{b}$ to the true distribution represented by a vector $\mathbf{x}$, which is determined by unfolding. The response matrix $\mathbf{A}$ describes the detector acceptance, contains all selection efficiencies and takes migration effects between bins into account. Additional background, not determined by the unfolding procedure and taken from external information, is represented by the vector $\mathbf{b}$.

An estimator $\hat{\mathbf{x}}$ of the true distribution $\mathbf{x}$ is obtained by unfolding the measured distribution $\mathbf{y}$. For the construction of $\hat{\mathbf{x}}$ additional assumptions, e.g. on the smoothness of the de-convoluted distribution (regularisation), and an additional constraint on the number of observed events are applied. In general $\hat{\mathbf{x}}$ is obtained by minimising a $\chi^{2}$ function given by:

$\chi^{2}(\hat{\mathbf{x}}, \tau, \mu):=\chi_{A}^{2}(\hat{\mathbf{x}})+\tau \cdot \chi_{L}^{2}(\hat{\mathbf{x}})+\mu \cdot \chi_{N}^{2}(\hat{\mathbf{x}})$.
This equation describes the minimisation of the unfolding problem $\chi_{A}^{2}(\hat{\mathbf{x}})$ with the two side conditions given by $\chi_{L}^{2}(\hat{\mathbf{x}})$ and $\chi_{N}^{2}(\hat{\mathbf{x}})$.

The actual minimisation problem is defined by the standard $\chi^{2}$ function:

$\chi_{A}^{2}(\hat{\mathbf{x}}):=\frac{1}{2}(\mathbf{y}-\mathbf{b}-\mathbf{A} \hat{\mathbf{x}})^{T} \mathbf{V}^{-1}(\mathbf{y}-\mathbf{b}-\mathbf{A} \hat{\mathbf{x}})$,

with $\mathbf{V}=\operatorname{cov}\left(y_{i}, y_{j}\right)$ being the covariance matrix of the data. This function minimises the deviation of the estimator $\mathbf{A} \hat{\mathbf{x}}$ from the measured, and background subtracted vector $\mathbf{y}-\mathbf{b}$.

The additional constraints are given by:

$\chi_{L}^{2}(\hat{\mathbf{x}}):=\hat{\mathbf{x}}^{T} \mathbf{L} \hat{\mathbf{x}}$,

$\chi_{N}^{2}(\hat{\mathbf{x}}):=\left(n_{\mathrm{obs}}-\sum_{j=1}^{m}(\mathbf{A} \hat{\mathbf{x}})_{j}\right)^{2}$,

with $\mathbf{L}$ being the regularisation matrix, $m$ the number of reconstructed bins and $n_{\text {obs }}$ the total number of observed events after background subtraction, which ensures that the total number of events is conserved. Both functions enter equation 13 with the parameters $\tau$ and $\mu$, where $\tau$ is often denoted as regularisation parameter and $\mu$ as Lagrange Multiplier.

The $\chi_{L}^{2}(\hat{\mathbf{x}})$ function is a measure for the smoothness of the result. The matrix $L$ is chosen such that the second derivative of $\hat{\mathbf{x}}$ between bins describing beauty production is minimised. The regularisation parameter $\tau$ determines the strength of the smoothness constraint. For the regularised unfolding $\tau$ is chosen such that the correlations of the covariance matrix of the unfolded distribution $\hat{\mathbf{x}}$ are minimised [78]. 


\section{References}

1. F.D. Aaron et al. (H1 Collaboration), DESY-12-059, Eur. Phys. J. C 72, 2047 (2012). arXiv:1205.2495

2. A. Aktas et al. (H1 Collaboration), Eur. Phys. J. C 47, 597 (2006). hep-ex/0605016

3. A. Aktas et al. (H1 Collaboration), Phys. Lett. B 621, 56 (2005). hep-ex/0503038

4. A. Aktas et al. (H1 Collaboration), Eur. Phys. J. C 41, 453 (2005). hep-ex/0502010

5. C. Adloff et al. (H1 Collaboration), Phys. Lett. B 467, 156 (1999). Erratum-ibid. B 518, 331 (2001). hep-ex/9909029

6. H. Abramowicz et al. (ZEUS Collaboration), Eur. Phys. J. C 71, 1659 (2011). arXiv:1104.5444

7. S. Chekanov et al. (ZEUS Collaboration), J. High Energy Phys. 0904, 133 (2009). arXiv:0901.2226

8. S. Chekanov et al. (ZEUS Collaboration), J. High Energy Phys. 0902, 032 (2009). arXiv:0811.0894

9. S. Chekanov et al. (ZEUS Collaboration), Phys. Rev. D 78, 072001 (2008). arXiv:0805.4390

10. S. Chekanov et al. (ZEUS Collaboration), Eur. Phys. J. C 50, 299 (2007). hep-ex/0609050

11. S. Chekanov et al. (ZEUS Collaboration), Phys. Rev. D 70, 012008 (2004). Erratum-ibid., D 74, 059906 (2006). hep-ex/0312057

12. J. Breitweg et al. (ZEUS Collaboration), Eur. Phys. J. C 18, 625 (2001). hep-ex/0011081

13. S. Frixione, M.L. Mangano, P. Nason, G. Ridolfi, Adv. Ser. Dir. High Energy Phys. 15, 609 (1998). hep-ph/9702287

14. S. Frixione, P. Nason, G. Ridolfi, Nucl. Phys. B 454, 3 (1995). hep-ph/9506226

15. S. Frixione, M.L. Mangano, P. Nason, G. Ridolfi, Phys. Lett. B 348, 633 (1995). hep-ph/9412348

16. A. Geiser, Nucl. Phys. B, Proc. Suppl. 184, 189 (2008)

17. H. Abramowicz et al. (ZEUS collaboration), Eur. Phys. J. C 71, 1573 (2011). arXiv:1101.3692

18. H. Abramowicz et al. (ZEUS collaboration), Eur. Phys. J. C 69, 347 (2010). arXiv: 1005.3396

19. S. Chekanov et al. (ZEUS Collaboration), Eur. Phys. J. C 65, 65 (2010). arXiv:0904.3487

20. S. Chekanov et al. (ZEUS Collaboration), Phys. Lett. B 599, 173 (2004). hep-ex/0405069

21. F.D. Aaron et al. (H1 Collaboration), Eur. Phys. J. C 71, 1509 (2011). arXiv:1008.1731

22. F.D. Aaron et al. (H1 Collaboration), Eur. Phys. J. C 65, 89 (2010). arXiv:0907.2643

23. A. Aktas et al. (H1 Collaboration), Eur. Phys. J. C 45, 23 (2006). hep-ex/0507081

24. A. Aktas et al. (H1 Collaboration), Eur. Phys. J. C 40, 349 (2005). hep-ex/0411046

25. T. Sjöstrand, L. Lönnblad, S. Mrenna (PYTHIA 6.2), LU TP 0121. hep-ph/0108264

26. T. Sjöstrand et al., Comput. Phys. Commun. 135, 238 (2001). hep-ph/0010017

27. H. Jung, G.P. Salam (CASCADE 2.0), Eur. Phys. J. C 19, 351 (2001). hep-ph/0012143

28. H. Jung, Comput. Phys. Commun. 143, 100 (2002). hep-ph/ 0109102

29. M. Hansson, H. Jung, in Proceedings of "XI International Workshop on Deep Inelastic Scattering (DIS 2003)”, ed. by V.T. Kim, L.N. Lipatov (2003), p. 488. hep-ph/0309009

30. H. Jung (RAPGAP 3.1), Comput. Phys. Commun. 86, 147 (1995)

31. J. Pumplin et al., J. High Energy Phys. 0207, 012 (2002). hep-ph/0201195

32. V.N. Gribov, L.N. Lipatov, Yad. Fiz. 15, 781 (1972). Sov. J. Nucl. Phys., 15, 438 (1972)
33. G. Altarelli, G. Parisi, Nucl. Phys. B 126, 298 (1977)

34. Y.L. Dokshitzer, Sov. Phys. JETP 46, 641 (1977). Zh. Eksp. Teor. Fiz., 73, 1216 (1977)

35. G.A. Schuler, T. Sjöstrand, Phys. Lett. B 376, 193 (1996). hep-ph/9601282

36. H. Jung, in Proceedings of "XII International Workshop in DeepInelastic Scattering (DIS 2004)”, ed. by D. Bruncko, J. Ferencei, P. Stríženec (2004), p. 299. hep-ph/0411287

37. M. Ciafaloni, Nucl. Phys. B 296, 49 (1988)

38. S. Catani, F. Fiorani, G. Marchesini, Phys. Lett. B 234, 339 (1990)

39. S. Catani, F. Fiorani, G. Marchesini, Nucl. Phys. B 336, 18 (1990)

40. G. Marchesini, Nucl. Phys. B 445, 49 (1995). hep-ph/9412327

41. B. Andersson, G. Gustafson, B. Söderberg, Z. Phys. C 20, 317 (1983)

42. B. Andersson, G. Gustafson, G. Ingelman, T. Sjöstrand, Phys. Rep. 97, 31 (1983)

43. M.G. Bowler, Z. Phys. C 11, 169 (1981)

44. R. Brun et al. (GEANT 3), CERN-DD/EE/84-1 (1987)

45. M. Glück, E. Reya, A. Vogt, Eur. Phys. J. C 5, 461 (1998)

46. I. Abt et al. (H1 Collaboration), Nucl. Instrum. Methods A $\mathbf{3 8 6}$, 310 (1997)

47. I. Abt et al. (H1 Collaboration), Nucl. Instrum. Methods A $\mathbf{3 8 6}$, 348 (1997)

48. R.D. Appuhn et al., Nucl. Instrum. Methods A 386, 397 (1997)

49. D. Pitzl et al., Nucl. Instrum. Methods A 454, 334 (2000). hep-ex/ 0002044

50. J. Becker et al., Nucl. Instrum. Methods A 586, 190 (2008). physics/0701002

51. B. Andrieu et al. (H1 Calorimeter Group), Nucl. Instrum. Methods A 336, 460 (1993)

52. B. Andrieu et al. (H1 Calorimeter Group), Nucl. Instrum. Methods A 350, 57 (1994)

53. B. Andrieu et al. (H1 Calorimeter Group), Nucl. Instrum. Methods A 336, 499 (1993)

54. F.D. Aaron et al. (H1 Collaboration), DESY-12-062, Eur. Phys. J. C, submitted. arXiv: 1205.2448

55. A. Baird et al., IEEE Trans. Nucl. Sci. 48, 1276 (2001). hep-ex/ 0104010

56. D. Meer et al., IEEE Trans. Nucl. Sci. 49, 357 (2002). hep-ex/0107010

57. A. Schöning (H1 Collaboration), Nucl. Instrum. Methods A 518, 542 (2004)

58. N. Berger et al., in IEEE Nuclear Science Symposium Conference Record, vol. 3 (2004), p. 1976

59. A. Schöning (H1 Collaboration), Nucl. Instrum. Methods A 566, $130(2006)$

60. A.W. Jung et al., in Proceedings of "15th IEEE-NPSS Real-Time Conference" (2007)

61. M. Sauter, Measurement of beauty photoproduction at threshold using di-electron events with the $\mathrm{H} 1$ detector at HERA. Ph.D. thesis, ETH Zürich (2009), Diss. ETH No. 18652 and DESY-THESIS-2009-047 (available at http://www-h1.desy.de/ publications/theses_list.html)

62. L. Caminada, Implementation of a trigger for the decay $b \rightarrow e X$ on the third trigger level at the H1 experiment. Diploma thesis, ETH Zürich (2006), ETHZ-IPP RP-2006-06 (available at http://www-h1.desy.de/publications/theses_list.html)

63. B. Olivier et al., Nucl. Instrum. Methods A 641, 58 (2011)

64. A. Hoecker et al., in Proceedings of "XI International Workshop on Advanced Computing and Analysis Techniques in Physics Research", PoS (ACAT) 040 (2007). physics/0703039

65. E. Hennekemper, Simulation and calibration of the specific energy loss of the central jet chambers of the $\mathrm{H} 1$ detector and measurement of the inclusive $D^{* \pm}$ meson cross section in photoproduction at HERA. Ph.D. thesis, University Heidelberg (2011), HD-KIP-11-68 (available at http://www-h1.desy.de/publications/ theses_list.html) 
66. F.D. Aaron et al. (H1 Collaboration), Eur. Phys. J. C 63, 625 (2009). arXiv:0904.0929

67. P. Bruel, Recherche d'interactions au-delà du Modèle Standard à HERA. Ph.D. thesis, L'Université Paris XI Orsay (1998) (available at http://www-h1.desy.de/publications/theses_list.html)

68. M. Peez, Recherche de déviations au Modèle Standard dans les processus de grande énergie transverse sur le collisionneur électron-proton HERA. Ph.D. thesis, Université de Lyon (2003), DESY-THESIS-2003-023 (available at http://www-h1.desy.de/ publications/theses_list.html)

69. B. Portheault, Première mésure des sections efficaces de courant chargé et neutre avec le faisceau de positrons polarisés HERA II et analyses QCD-électrofaibles. Ph.D. thesis, Université Paris XI (2005), LAL-05-05 (available at http://www-h1.desy.de/publications/theses_list.html)

70. S. Hellwig, Untersuchung der $D^{*}-\pi_{\text {slow }}$ Double Tagging Methode in Charmanalysen. Diploma thesis, Univ. Ham- burg (2004) (available at http://www-h1.desy.de/publications/ theses_list.html)

71. K. Nakamura et al. (Particle Data Group), J. Phys. G 37, 075021 (2010)

72. F.D. Aaron et al. (H1 Collaboration), Eur. Phys. J. C 59, 589 (2009). arXiv:0808.1003

73. F.D. Aaron et al. (H1 Collaboration), Eur. Phys. J. C 68, 401 (2010). arXiv:1002.0234

74. F.D. Aaron et al. (H1 Collaboration), Eur. Phys. J. C 72, 1995 (2012). arXiv:1203.1170

75. E. Barberio, Z. Was, Comput. Phys. Commun. 79, 291 (1994)

76. G.D. Lafferty, T.R. Wyatt, Nucl. Instrum. Methods A 355, 541 (1995)

77. S. Schmitt. TUnfold 16.1, available at http://www.desy.de/ sschmitt/tunfold.html arXiv:1205.6201

78. V. Blobel, in Proceedings of "Conference on Advanced Statistical Techniques in Particle Physics", ed. by M.R. Whalley, L. Lyons, Durham, England (2002), p. 258. hep-ex/0208022 